\title{
Realization of Point Planar Elastic Behaviors Using Revolute Joint Serial Mechanisms Having Specified Link Lengths
}

\author{
Shuguang Huang, ${ }^{\mathrm{a}, *}$ Joseph M. Schimmels ${ }^{\mathrm{a}}$ \\ ${ }^{a}$ Department of Mechanical Engineering, Marquette University, Milwaukee, WI 53201, USA
}

\begin{abstract}
This paper presents methods for the realization of $2 \times 2$ translational compliance matrices using serial mechanisms having only revolute joints, each with selectable compliance. The link lengths of the mechanism and the location of the compliant frame relative to the mechanism base are arbitrary but specified. The realizability of a given compliant behavior is investigated, and necessary and sufficient conditions for the realization of a given compliance with a given mechanism are obtained. These realization conditions are interpreted in terms of geometric relationships among the joints. We show that, for an appropriately sized 3R serial mechanism, any single $2 \times 2$ compliance matrix can be realized by properly choosing the joint compliances and the mechanism configuration. Requirements on mechanism geometry to realize every particle planar elastic behavior at a given location just by changing the mechanism configuration are also identified.
\end{abstract}

Keywords: Compliance realization, compliant mechanism, stiffness and compliance synthesis

\section{Introduction}

In order for robots to achieve human-like dexterity in manipulation tasks, a means for regulating interaction force is needed, especially when geometric conflict exists between the robot and its environment. One approach to force regulation is to select or control the robot passive compliance. Passive compliance allows for high speed interaction. Desirable robot passive compliance can be attained by: 1) designing compliant end-of-arm tooling, or 2) designing compliance into the arm itself.

Any desired compliant behavior can be realized through the proper design of an end-effector mounted compliant wrist. It is known that for any specified elastic behavior, an infinite number of parallel [1, 2] or serial [3, 4] mechanisms having springs at each joint can achieve the desired behavior. Proper compliant wrist design involves selecting the geometry and configuration of the mechanism and selecting the spring rates at each joint. The limitation of this approach is that the mechanism realizes a single specified compliance. If the robot task changes or the desired compliance within the task changes, an entirely different compliant wrist would likely be needed for its realization.

Several strategies exist for designing compliance into the manipulator itself. Series elastic actuators (SEAs) [5], in which passive compliance is provided between the motor and the connected link can be used to provide a selected amount of compliance in each joint. Variable stiffness actuators (VSAs) [6] are similar, but they allow joint compliance to be changed in real time. This ability is important in robot tasks that require continuously changing compliant behaviors, such as when the nature of the task changes, the task itself changes, or the task environment changes (especially when people are in the robot workspace).

VSAs enlarge the space of elastic behavior that can be achieved by a robot by changing each joint stiffness. If the manipulator is kinematically redundant, the set of achievable elastic behaviors is increased

\footnotetext{
* Corresponding author

Email addresses: huangs@marquette.edu (Shuguang Huang), joseph.schimmels@marquette.edu (Joseph M. Schimmels)
} 
further. A much larger set of compliances can be attained by adjusting both the joint stiffnesses and the manipulator configuration, without ever changing the endpoint location of the robot.

In some manipulation tasks, such as turning a crank or opening a door, the interaction torque is not important. Since only the relationship between force and translation is important, the interaction can be modeled as point contact, and the compliant behavior can be modeled as an elastically suspended particle, i.e., not an elastically suspended rigid body. This paper presents methods for the evaluation and realization of planar translational elastic behaviors using serial mechanisms having only revolute joints, each with selectable compliance. An example of the type of mechanism considered is illustrated in Fig. 1 . The mechanisms considered are arbitrary but have known link lengths.

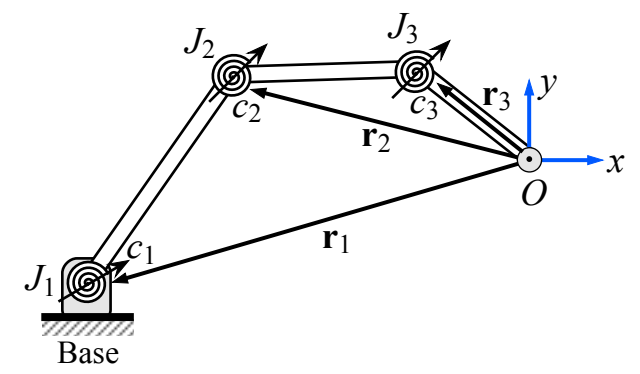

Figure 1: A 3R serial compliant mechanism with variable stiffness actuators.

\subsection{Related Work}

Spatial elastic behavior has been been analyzed for more than a century. Screw theory [7, 8, 9, 10, 11. and Lie groups [12] have been used to analyze and characterize spatial linear elastic behavior (represented by a $6 \times 6$ symmetric stiffness matrix $\mathbf{K}$ or compliance matrix $\mathbf{C}$ ).

More recent work has addressed the realization of spatial elastic behavior through the design of passive compliant mechanisms. Previously, the bounds of elastic behaviors achieved with simple parallel mechanisms [1] and with simple serial mechanisms [3] have been identified. A simple parallel mechanism contains only line springs and torsional springs, and a simple serial mechanism contains only prismatic and revolute joints. Synthesis procedures have been developed [1, 3, 13, 14, to realize any elastic behaviors within these bounds. Geometry based screw theory approaches for the realization of an arbitrary realizable stiffness matrix have also been developed [15, 16].

The synthesis of an arbitrary spatial stiffness matrix with a parallel system has also been addressed [2]. It was shown 2 that in order to realize an arbitrary stiffness, screw springs that couple the elastic behavior in translation and rotation in the same direction must be used. The eigen-structure of a stiffness was analyzed using the eigenscrew decomposition of the stiffness matrix 17. Based on the eigenstiffness analysis, a procedure to calculate the minimum number of screw springs required to realize a specified stiffness matrix in a parallel system was identified [18. A procedure has been developed [19] to realize an arbitrary spatial stiffness using the minimum numbers of screw springs and simple springs.

This previous work has focused on the realization of a single compliance with a mechanism of unspecified geometry at a unspecified configuration. When the desired compliance is changed, a different mechanism with a new configuration must be used. Very little work has addressed: 1) the ability of a single specified mechanism to realize an arbitrary compliance, and 2) the identification of the space of realizable compliances that can be achieved with a mechanism having specified link lengths.

The inability of a $2 \mathrm{R}$ compliant joint manipulator to realize general particle planar compliance was noted in 20]. It was also noted that, for a $3 \mathrm{R}$ manipulator, the surjectivity of the mapping from $2 \times 2$ Cartesian space to joint space compliance is not guaranteed. These limitations were identified as motivation for using optimization (using various norms as objective functions) for approximating the behavior of a targeted Cartesian compliance matrix. More recently, the ability of a manipulator to realize a specified point planar compliance using 3R serial mechanisms with known link lengths are addressed [21]. Optimization was used 
to identify the combination of mechanism configuration and joint stiffnesses that minimizes the deviation of the attainable Cartesian compliance from a targeted value. The proximity of an optimized compliance to a targeted compliance (measured using the matrix Frobenius norm) was characterized for a set of configurations of the redundant manipulator and for a set of compliant behaviors using a "stiffability map."

The major limitations of using optimization for the realization problem are that: 1) optimization does not ensure that any specified compliance can be achieved even with a large number of redundant joints; 2) the optimal solution may not preserve the desired compliant behavior of the original compliance; and 3) the results obtained from optimization provide little physical insight into the limits of passive compliance realization.

In more recent work [22, 23, 24, the realization of isotropic compliance in the Euclidian spaces $E(2)$ and $E(3)$ with robotic mechanisms has been addressed. Equations relating the Cartesian compliance entries to the joint compliance and the mechanism geometry were developed. In these papers, the joint compliances are calculated by solving these equations. The approach applies to a general serial mechanism with revolute and prismatic joints. However, the existence of a physical solution $\left(k_{i} \geq 0\right)$ to the equations is not guaranteed, and, for many configurations, a solution cannot exist. The set of mechanism configurations for which a solution exists was not identified.

This paper addresses the guaranteed realization of planar translational elastic behaviors with a serial mechanism having specified link lengths. The serial mechanisms considered have only revolute joints each loaded with a joint compliance. The compliance behaviors considered are arbitrary in $E(2)$. This work is motivated by the need for a single manipulator to realize any selected planar compliance at any selected endpoint. The paper presents the theory and methods to design a single manipulator that is able to achieve all compliances by changing its configuration and joint compliances. It also presents the theory and methods for the selection of mechanism configuration and joint stiffnesses needed to achieve any specified elastic behavior. The conditions for the realization of a given compliance are interpreted in terms of geometric relationships among the mechanism joints. The main contributions of the paper are:

1. Necessary and sufficient conditions for a mechanism to realize an arbitrarily given compliance at a given configuration are identified;

2. Synthesis procedures for the realization of a realizable compliance at a given location are developed;

3. Classes of mechanisms that facilitate compliance realization are identified. Necessary and sufficient conditions on the mechanism link lengths that ensure that all compliant behaviors can be realized are identified.

\subsection{Technical Background}

Consider a planar serial mechanism having $n$ joints, $J_{i}$, with joint compliance $c_{i}>0$ and joint twists $\mathbf{t}_{i}$. Then the compliance matrix $\mathbf{C}$ at the mechanism endpoint is a symmetric positive semidefinite (PSD) matrix that can be expressed [4] as:

$$
\mathbf{C}=c_{1} \mathbf{t}_{1} \mathbf{t}_{1}^{T}+c_{2} \mathbf{t}_{2} \mathbf{t}_{2}^{T}+\cdots+c_{n} \mathbf{t}_{n} \mathbf{t}_{n}^{T}
$$

where the twists $\mathbf{t}_{i}$ are described relative to the compliance frame (the reference frame where the compliance matrix is specified). Each compliant joint provides a rank-1 PSD component:

$$
\mathbf{C}_{i}=c_{i} \mathbf{t}_{i} \mathbf{t}_{i}^{T}
$$

It is known that decomposition (1) is not unique; different decompositions yield different mechanism geometries and configurations that each realizes the given compliance. Thus, decomposition (1) cannot be applied directly to a given mechanism with specified link lengths. A new method that considers mechanism geometry (fixed link lengths) to obtain an appropriate decomposition (1) is needed.

For the mechanism illustrated in Fig. 1, in which the compliance matrix is expressed in the frame at point $O$, each joint twist is given as:

$$
\mathbf{t}_{i}=\mathbf{r}_{i} \times \mathbf{k}
$$


where $\mathbf{r}_{i}$ is the position vector from $O$ to joint $i$ and $\mathbf{k}$ is the unit vector perpendicular to the plane. Thus, the joint twist $\mathbf{t}_{i}$ is determined by the position vector $\mathbf{r}_{i}$.

To realize a given compliance with a given mechanism, the mechanism configuration and the joint compliance $c_{i}$ must be adjusted so that Eq. (1) is satisfied.

For a suspended particle, the compliance is a $2 \times 2$ PSD matrix. If the position vector for joint- $i$ is:

$$
\mathbf{r}_{i}=\left[\begin{array}{l}
x_{i} \\
y_{i}
\end{array}\right]
$$

then the corresponding joint twist, perpendicular to $\mathbf{r}_{i}$, is:

$$
\mathbf{t}_{i}=\left[\begin{array}{r}
y_{i} \\
-x_{i}
\end{array}\right]
$$

From Eq. (2), it can be seen that $\mathbf{t}_{i}$ and $-\mathbf{t}_{i}$ yield the same joint compliance $\mathbf{C}_{i}$. Thus, $\mathbf{t}_{i}$ can be obtained by rotating $\mathbf{r}_{i}$ either clockwise or counterclockwise about the coordinate frame origin. Mathematically,

$$
\mathbf{t}_{i}= \pm \Omega \mathbf{r}_{i}
$$

where $\boldsymbol{\Omega}$ is a $2 \times 2$ anti-symmetric matrix associated with a vector cross-product, given as:

$$
\boldsymbol{\Omega}=\left[\begin{array}{rr}
0 & 1 \\
-1 & 0
\end{array}\right]
$$

It is known that the space of PSD matrices is a cone. All rank-1 PSD matrices lie on the boundary of the cone. Thus, each joint compliance matrix $\mathbf{C}_{i}$ in Eq. (2) is a point on the boundary of the PSD cone. If $c_{i}$ is allowed to vary in the range $0<c_{i}<\infty, \mathbf{C}_{i}$ represents a ray in the $2 \times 2$ PSD space, an edge of the PSD cone.

If a desired $2 \times 2$ PSD compliance matrix $\mathbf{C}$ is to be realized at the endpoint of a redundant $3 \mathrm{R}$ mechanism in which each joint has selectable compliance (as illustrated in Fig. 1), then, for a specified configuration, the three joint twists $\mathbf{t}_{i}$ are known. The desired $\mathbf{C}$ can be realized if and only if there exist non-negative coefficients $c_{i}$ such that

$$
\mathbf{C}=c_{1} \mathbf{t}_{1} \mathbf{t}_{1}^{T}+c_{2} \mathbf{t}_{2} \mathbf{t}_{2}^{T}+c_{3} \mathbf{t}_{3} \mathbf{t}_{3}^{T}
$$

For any $2 \times 2$ symmetric matrix $\mathbf{A}=\left[a_{i j}\right]$, only three entries are independent, therefore, $\mathbf{A}$ can be represented by a 3 -vector $\hat{A}=\left[a_{11}, a_{12}, a_{22}\right]^{T}$. With this operation, Eq. (5) can be written in vector form as:

$$
\hat{C}=c_{1} \hat{T}_{1}+c_{2} \hat{T}_{2}+c_{3} \hat{T}_{3}=\left[\hat{T}_{1}, \hat{T}_{2}, \hat{T}_{3}\right]\left[\begin{array}{l}
c_{1} \\
c_{2} \\
c_{3}
\end{array}\right]
$$

where $\hat{C}$ is the 3 -vector representation of $\mathbf{C}$; and $\hat{T}_{i}$ is the 3 -vector representation of $\mathbf{t}_{i} \mathbf{t}_{i}^{T}$. If we denote

$$
\hat{\mathbf{T}}=\left[\hat{T}_{1}, \hat{T}_{2}, \hat{T}_{3}\right], \quad \mathbf{c}=\left[c_{1}, c_{2}, c_{3}\right]^{T}
$$

then,

$$
\mathbf{c}=\hat{\mathbf{T}}^{-1} \hat{C}
$$

Thus, $\mathbf{C}$ can be realized at the given configuration if and only if $\mathbf{c} \geq 0$.

In the $2 \times 2$ PSD space, the condition $\mathbf{c} \geq 0$ indicates that $\mathbf{C}$ must be inside the polyhedral convex cone defined by the three edges $\mathbf{t}_{1} \mathbf{t}_{1}^{T}, \mathbf{t}_{2} \mathbf{t}_{2}^{T}$, and $\mathbf{t}_{3} \mathbf{t}_{3}^{T}$ as shown in Fig. 2. Because the manipulator is redundant, many configurations yield the same manipulator endpoint. When the mechanism changes configuration, the edge locations on the PSD cone's boundary also change. The solution is tractable because the dimension 


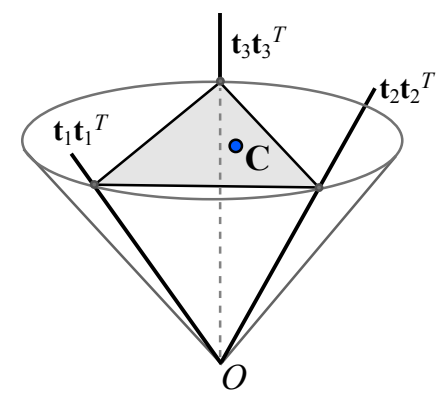

Figure 2: Realizable condition for a given compliance $\mathbf{C}$ with a $3 \mathrm{R}$ mechanism having a specified configuration. $\mathbf{C}$ must be inside of a polyhedral cone with edges $\mathbf{t}_{1} \mathbf{t}_{1}^{T}, \mathbf{t}_{2} \mathbf{t}_{2}^{T}$, and $\mathbf{t}_{3} \mathbf{t}_{3}^{T}$.

of the problem is the same as that of the manipulator. Although Eq. (6) can be used to determine the realizability of $\mathbf{C}$ for a given mechanism configuration, it does not provide insight into the geometric relations among the joints that are required for compliance matrix realization (i.e, to ensure that $\mathbf{C}$ is contained within the polyhedral convex cone bounded by its edges). For example, if $J_{1}$ and $J_{2}$ are specified, the space of $J_{3}$ locations that allows $\mathbf{C}$ to be realized is unknown. Also, Eq. (6) cannot be used to assess whether a mechanism can realize every compliance at a given location in the workspace.

\subsection{Overview}

This paper presents means to analyze and realize planar translational elastic behaviors using revolute joint serial mechanisms. The means for analysis and synthesis are based on the geometry of the realizable compliance matrix space and the geometry of the mechanism. This approach provides physical insight into elastic mechanism behavior. The ability of a single mechanism with specified link lengths to realize all compliance behaviors at a given end-point location is also investigated. In this paper, we assume that the range of joint compliances is $0<c_{i}<\infty$. Joint compliances can be obtained via one of the following approaches: 1) providing an appropriate (passive) spring at each joint; 2) providing an appropriate stiffness serial elastic actuator (SEA) at each joint; or 3) providing a variable stiffness actuator (VSA) at each joint.

Section 2 identifies the limits of a $2 \mathrm{R}$ mechanism to realize a specified $2 \times 2$ compliance matrix. A necessary and sufficient condition for a compliance to be realized by a $2 \mathrm{R}$ mechanism is identified. Section 3 presents an approach to realize a given compliance with a $3 \mathrm{R}$ mechanism based on mechanism geometry. The method then is extended to $n$ - $\mathrm{R}$ mechanisms. Section 4 addresses the ability of a single mechanism to realize all compliances at a given location just by changing the mechanism configuration and/or joint stiffnesses. A necessary and sufficient condition on the mechanism geometry to realize all compliances is identified. Synthesis procedures to achieve a realizable compliance are presented in Section 5. A numerical example illustrating the synthesis procedures for a given mechanism is provided in Section 6 . Finally, a brief discussion and summary are presented in Section 7.

\section{Limits of a $2 R$ Mechanism}

To realize a full-rank $2 \times 2$ compliance matrix, a mechanism having at least two compliant joints is required. Here, the limitation of a $2 \mathrm{R}$ serial mechanism with fixed link lengths and configuration is considered. A necessary and sufficient condition for a specified compliance to be realized with the mechanism is identified. These results allow the characterization of the realizable space of compliances in terms of the geometry of the mechanism.

Consider the 2R serial mechanism shown in Fig. 3. The mechanism endpoint $O$ is the location of interest (where the compliance frame is located) and $\mathbf{r}_{1}$ and $\mathbf{r}_{2}$ are the position vectors indicating the locations of joint- 1 and joint- 2 relative to $O$. If the position of $O$ relative to $J_{1}$ (the location in the workspace) is specified, the configuration of the mechanism cannot change, and the two position vectors $\mathbf{r}_{1}$ and $\mathbf{r}_{2}$ cannot vary. 


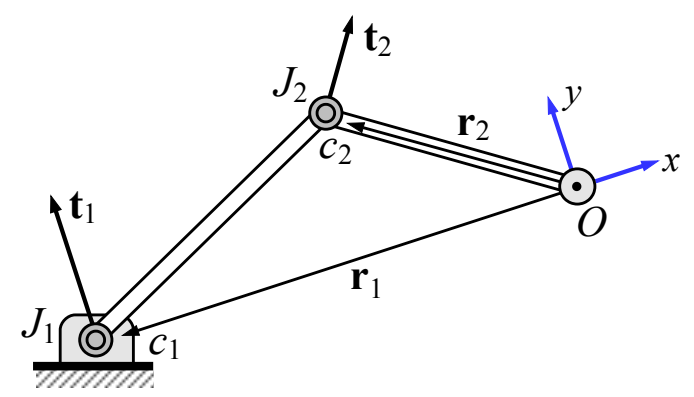

Figure 3: A 2R mechanism. Each joint twist $\mathbf{t}_{i}$ is perpendicular to the position vector $\mathbf{r}_{i}$ relative to the compliance frame $O x y$.

The space of realizable elastic behaviors is given by:

Proposition 1: A compliance matrix $\mathbf{C}$ can be realized with a $2 \mathrm{R}$ mechanism in a given configuration if and only if:

$$
\mathbf{r}_{1}^{T} \mathbf{C} \mathbf{r}_{2}=0
$$

Proof. If $\mathbf{C}$ is realized by the mechanism, then, by Eq. (1), there exist positive constants, the joint compliances, $c_{1}$ and $c_{2}$, that satisfy

$$
\mathbf{C}=c_{1} \mathbf{t}_{1} \mathbf{t}_{1}^{T}+c_{2} \mathbf{t}_{2} \mathbf{t}_{2}^{T}
$$

Since $\mathbf{r}_{1} \perp \mathbf{t}_{1}$ and $\mathbf{r}_{2} \perp \mathbf{t}_{2}$, then $\mathbf{r}_{1}^{T} \mathbf{t}_{1}=0$ and $\mathbf{r}_{2}^{T} \mathbf{t}_{2}=0$. Thus,

$$
\begin{aligned}
\mathbf{r}_{1}^{T} \mathbf{C r}_{2} & =\mathbf{r}_{1}^{T}\left(c_{1} \mathbf{t}_{1} \mathbf{t}_{1}^{T}+c_{2} \mathbf{t}_{2} \mathbf{t}_{2}^{T}\right) \mathbf{r}_{2} \\
& =c_{1}\left(\mathbf{r}_{1}^{T} \mathbf{t}_{1}\right)\left(\mathbf{t}_{1}^{T} \mathbf{r}_{2}\right)+c_{2}\left(\mathbf{r}_{1}^{T} \mathbf{t}_{2}\right)\left(\mathbf{t}_{2}^{T} \mathbf{r}_{2}\right)=0,
\end{aligned}
$$

which proves that Eq. (7) is a necessary condition.

To prove that the condition is also sufficient, we show that $\mathbf{C}$ can always be expressed by a known positive combination of $\mathbf{t}_{1} \mathbf{t}_{1}^{T}$ and $\mathbf{t}_{2} \mathbf{t}_{2}^{T}$ if Eq. (7) is satisfied.

Suppose $\mathbf{r}_{1}^{T} \mathbf{C} \mathbf{r}_{2}=0$, then $\mathbf{C r}_{2} \perp \mathbf{r}_{1}$. Due to the symmetry of matrix $\mathbf{C}, \mathbf{r}_{2}^{T} \mathbf{C} \mathbf{r}_{1}=0$, and $\mathbf{C r}_{1} \perp \mathbf{r}_{2}$. Using this and Eq. (3), $\mathbf{t}_{1} \| \mathbf{C r}_{2}$ and $\mathbf{t}_{2} \| \mathbf{C r}_{1}$. Thus there exist constants $\lambda$ and $\beta$ such that $\lambda \mathbf{t}_{1}=\mathbf{C r}_{2}$, and $\beta \mathbf{t}_{2}=\mathbf{C r}_{1}$. Then $\mathbf{t}_{1}$ and $\mathbf{t}_{2}$ can be expressed as:

$$
\mathbf{t}_{1}=\frac{1}{\lambda} \mathbf{C r}_{2}, \quad \mathbf{t}_{2}=\frac{1}{\beta} \mathbf{C r}_{1} .
$$

It can be shown that a matrix $\tilde{\mathbf{C}}$ constructed as:

$$
\tilde{\mathbf{C}}=\frac{\lambda^{2}}{\mathbf{r}_{2}^{T} \mathbf{C r}_{2}} \mathbf{t}_{1} \mathbf{t}_{1}^{T}+\frac{\beta^{2}}{\mathbf{r}_{1}^{T} \mathbf{C r}_{1}} \mathbf{t}_{2} \mathbf{t}_{2}^{T}
$$

must be equal to $\mathbf{C}$.

This is shown by substituting Eq. (8) into Eq. (9) yielding

$$
\tilde{\mathbf{C}}=\frac{1}{\left(\mathbf{r}_{2}^{T} \mathbf{C r}\right)} \mathbf{C r}_{2} \mathbf{r}_{2}^{T} \mathbf{C}+\frac{1}{\left(\mathbf{r}_{1}^{T} \mathbf{C r}\right)} \mathbf{C r}_{1} \mathbf{r}_{1}^{T} \mathbf{C},
$$

then multiplying $\tilde{\mathbf{C}}$ by $\mathbf{r}_{1}$ from the right yields

$$
\begin{aligned}
\tilde{\mathbf{C}} \mathbf{r}_{1} & =\frac{1}{\left(\mathbf{r}_{2}^{T} \mathbf{C r} \mathbf{r}_{2}\right)} \mathbf{C r}_{2} \mathbf{r}_{2}^{T} \mathbf{C r}_{1}+\frac{1}{\left(\mathbf{r}_{1}^{T} \mathbf{C r}_{1}\right)} \mathbf{C r}_{1} \mathbf{r}_{1}^{T} \mathbf{C r}_{1} \\
& =\frac{1}{\left(\mathbf{r}_{2}^{T} \mathbf{C r} \mathbf{r}_{2}\right)} \mathbf{C r}_{2}\left(\mathbf{r}_{2}^{T} \mathbf{C r}_{1}\right)+\frac{1}{\left(\mathbf{r}_{1}^{T} \mathbf{C r}\right)} \mathbf{C r}_{1}\left(\mathbf{r}_{1}^{T} \mathbf{C r}_{1}\right) \\
& =\mathbf{C r}_{1} .
\end{aligned}
$$


Similarly, we have

$$
\tilde{\mathbf{C}} \mathbf{r}_{2}=\mathbf{C r}_{2}
$$

Therefore:

$$
\tilde{\mathbf{C}}\left[\mathbf{r}_{1}, \mathbf{r}_{2}\right]=\mathbf{C}\left[\mathbf{r}_{1}, \mathbf{r}_{2}\right] .
$$

Since $\mathbf{r}_{1}$ and $\mathbf{r}_{2}$ are two linearly independent vectors, then $\tilde{\mathbf{C}}=\mathbf{C}$. Thus, by choosing the joint compliance $c_{1}$ and $c_{2}$,

$$
c_{1}=\frac{\lambda^{2}}{\mathbf{r}_{2}^{T} \mathbf{C r}_{2}}, \quad c_{2}=\frac{\beta^{2}}{\mathbf{r}_{1}^{T} \mathbf{C r}_{1}}
$$

the compliance matrix $\mathbf{C}$ is realized by the mechanism with the given configuration. Note that $c_{i}>0$ since $\mathbf{C}$ is positive definite. This completes the proof.

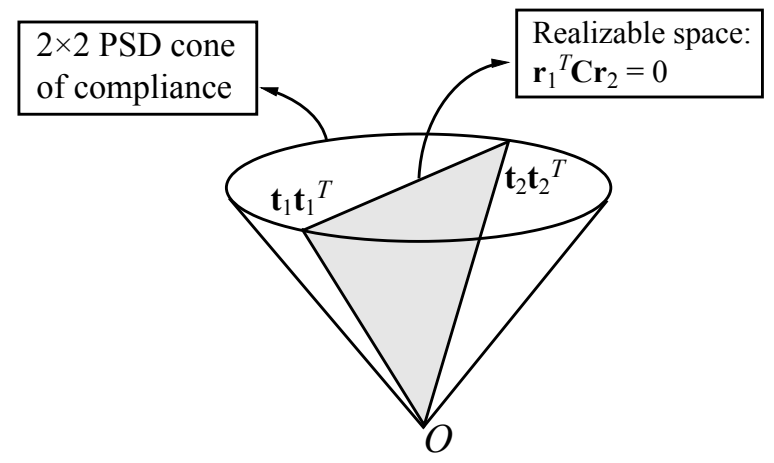

Figure 4: Realizable space of compliance matrices using a $2 \mathrm{R}$ mechanism having variable compliant joints and fixed configuration. The two edges of the subcone are the rank-1 matrices $\mathbf{t}_{1} \mathbf{t}_{1}^{T}$ and $\mathbf{t}_{2} \mathbf{t}_{2}^{T}$ associated with vectors $\mathbf{r}_{1}$ and $\mathbf{r}_{2}$.

Condition (7) is very restrictive. It is known that the collection of all point planar compliance matrices is a cone in the space of $2 \times 2$ symmetric matrices. Equation (7) represents a plane in this space. The realizable space of PSD matrices is a subcone determined by the intersection of the PSD cone and the plane defined by Eq. (7) (as shown in Fig. 4). The two edges of the subcone are the rays determined by $\mathbf{t}_{1} \mathbf{t}_{1}^{T}$ and $\mathbf{t}_{2} \mathbf{t}_{2}^{T}$ that are associated with the positions of the two joints, $J_{1}$ and $J_{2}$. For a given location of the compliance frame, the position of $J_{1}$ relative to $O, \mathbf{r}_{1}$, is specified. The ray $\mathbf{t}_{1} \mathbf{t}_{1}^{T}$ is constant and lies on the boundary of the PSD cone. For a given compliance $\mathbf{C}$, the plane passing through both edge $\mathbf{t}_{1} \mathbf{t}_{1}^{T}$ and compliance $\mathbf{C}$ must intersect the PSD cone at a ray. In order to realize $\mathbf{C}$, the second joint, $J_{2}$, must be positioned such that its joint compliance matrix is collinear with that ray.

The restriction on the space of realizable elastic behaviors can be described in terms of the mechanism geometry as illustrated in Fig. 5. Because the position of joint $J_{1}$ relative to point $O$ is specified, $\mathbf{r}_{1}$ is constant. By condition (7), vector $\mathbf{r}_{2}$ must be perpendicular to vector $\mathbf{C r}_{1}$. Let $l_{1}$ be the straight line passing through $O$ that is perpendicular to $\mathbf{C r}_{1}$. Condition (7) requires that $J_{2}$ be on line $l_{1}$. Thus, line $l_{1}$ represents the locus of all $J_{2}$ locations that will allow the given $\mathbf{C}$ to be realized by the mechanism.

Since the realizable space of a $2 \mathrm{R}$ mechanism is very limited (zero-measure), in order to realize an arbitrary compliance, serial mechanisms having at least three joints must be considered.

\section{Mechanisms with Three or More Compliant Joints}

The limits on the space of realizable compliances using a 2-joint mechanism are due to the fact that, once the position of suspended particle relative to the base location is specified, the configuration of the 


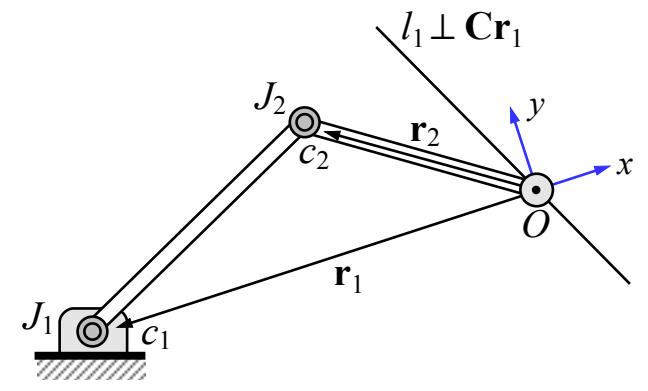

Figure 5: Limitation of the realization of a given compliance with a $2 \mathrm{R}$ mechanism. Joint $J_{2}$ must be on the line $l_{1} \perp \mathbf{C r}_{1}$ to realize the given compliance $\mathbf{C}$.

mechanism is fixed. In this section, mechanisms having three or more compliant joints are considered. Since the degree of freedom is increased, the mobility of the mechanism is no longer zero when the location of $O$ is specified. First, mechanisms having three joints are considered. Mathematical conditions on a compliance C to be realized with a mechanism having a given configuration are obtained. Next, the set of joint twists needed to realize a specified compliance are obtained. These conditions are then described in terms of the mechanism geometry. Alternate procedures to select the joint compliances based on the mechanism configuration are also provided. Then, the results are extended to mechanisms having more than three joints.

\subsection{Conditions for Compliance Realization}

Below, we develop a geometric approach in compliance matrix space that will later be used directly in physical 2-D space to determine the relationships among the three joints required to realize a specified compliance $\mathbf{C}$.

For joint-1 and the corresponding position vector $\mathbf{r}_{1}$, consider the two vectors defined by

$$
\tilde{\mathbf{t}}_{1}=\mathbf{C r}_{1}, \quad \tilde{\mathbf{r}}_{1}=\mathbf{\Omega} \mathbf{C r}_{1} .
$$

Then, because by Proposition 1,

$$
\mathbf{r}_{1}^{T} \mathbf{C} \tilde{\mathbf{r}}_{1}=\left(\mathbf{C r}_{1}\right)^{T} \boldsymbol{\Omega}\left(\mathbf{C r}_{1}\right)=0
$$

and $\tilde{\mathbf{t}}_{1}$ and $\tilde{\mathbf{r}}_{1}$ can be viewed as being associated with a nonexistent second joint needed to realize $\mathbf{C}$ with a 2R mechanism. The given compliance $\mathbf{C}$ can be expressed as the positive combination of $\mathbf{t}_{1} \mathbf{t}_{1}^{T}$ and $\tilde{\mathbf{t}}_{1} \tilde{\mathbf{t}}_{1}^{T}$, which means the compliance $\mathbf{C}$ is in the plane $P_{1}$ determined by the two rays $\mathbf{t}_{1} \mathbf{t}_{1}^{T}$ and $\tilde{\mathbf{t}}_{1} \tilde{\mathbf{t}}_{1}^{T}$ :

$$
P_{1}=\left\{\mathbf{Q} \in \mathcal{R}^{2 \times 2}: \mathbf{Q}=\mathbf{Q}^{T}, \mathbf{r}_{1}^{T} \mathbf{Q} \tilde{\mathbf{r}}_{1}=0\right\} .
$$

As illustrated in Fig. 6, plane $P_{1}$ is uniquely determined by the position of $J_{1}$ and the compliance matrix C.

If $\mathbf{C}$ is to be realized by a $3 \mathrm{R}$ mechanism, $\mathbf{C}$ must be within a polyhedral convex cone having edges $\mathbf{t}_{1} \mathbf{t}_{1}^{T}$, $\mathbf{t}_{2} \mathbf{t}_{2}^{T}$, and $\mathbf{t}_{3} \mathbf{t}_{3}^{T}$. Hence $\mathbf{C}_{2}=\mathbf{t}_{2} \mathbf{t}_{2}^{T}$ and $\mathbf{C}_{3}=\mathbf{t}_{3} \mathbf{t}_{3}^{T}$ must be on opposite sides of $P_{1}$, which means $\mathbf{r}_{1}^{T} \mathbf{C}_{2} \tilde{\mathbf{r}}_{1}$ and $\mathbf{r}_{1}^{T} \mathbf{C}_{3} \tilde{\mathbf{r}}_{1}$ must have opposite signs, which can be expressed as:

$$
\left(\mathbf{r}_{1}^{T} \mathbf{C}_{2} \tilde{\mathbf{r}}_{1}\right)\left(\mathbf{r}_{1}^{T} \mathbf{C}_{3} \tilde{\mathbf{r}}_{1}\right)<0 .
$$

Since $\boldsymbol{\Omega}^{T} \boldsymbol{\Omega}=\mathbf{I}$ (identity),

$$
\begin{aligned}
\mathbf{r}_{1}^{T} \mathbf{C}_{2} \tilde{\mathbf{r}}_{1} & =\mathbf{r}_{1}^{T}\left(\mathbf{t}_{2} \mathbf{t}_{2}^{T}\right) \boldsymbol{\Omega} \mathbf{C r}_{1}=\mathbf{r}_{1}^{T}\left(\boldsymbol{\Omega} \mathbf{r}_{2} \mathbf{r}_{2}^{T} \boldsymbol{\Omega}^{T}\right) \boldsymbol{\Omega} \mathbf{C r}_{1} \\
& =\mathbf{r}_{1}^{T}\left(\boldsymbol{\Omega} \mathbf{r}_{2} \mathbf{r}_{2}^{T}\right) \mathbf{C r}_{1}
\end{aligned}
$$




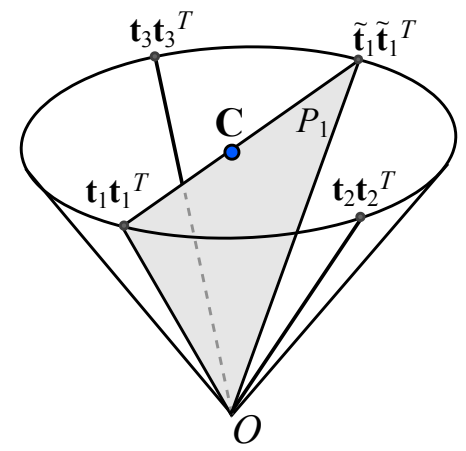

Figure 6: Condition to realize a given compliance $\mathbf{C}$. Plane $P_{1}$ is determined by $\mathbf{C}$ and the joint compliance of $J_{1}$, $\mathbf{t}_{1} \mathbf{t}_{1}^{T}$. To realize $\mathbf{C}$, plane $P_{1}$ must separate the joint compliances associated with joints $J_{2}$ and $J_{3}, \mathbf{t}_{2} \mathbf{t}_{2}^{T}$ and $\mathbf{t}_{3} \mathbf{t}_{3}^{T}$.

Similarly,

$$
\mathbf{r}_{1}^{T} \mathbf{C}_{3} \tilde{\mathbf{r}}_{1}=\mathbf{r}_{1}^{T}\left(\boldsymbol{\Omega} \mathbf{r}_{3} \mathbf{r}_{3}^{T}\right) \mathbf{C r}_{1}
$$

Thus Eq. (12) can be expressed as:

$$
\left(\mathbf{r}_{1}^{T} \boldsymbol{\Omega} \mathbf{r}_{2} \mathbf{r}_{2}^{T} \mathbf{C r}_{1}\right)\left(\mathbf{r}_{1}^{T} \boldsymbol{\Omega} \mathbf{r}_{3} \mathbf{r}_{3}^{T} \mathbf{C} \mathbf{r}_{1}\right)<0 .
$$

The above inequality is a necessary condition. In order to obtain sufficient conditions, the compliance matrix edges associated with the other two joints, $\mathbf{t}_{2} \mathbf{t}_{2}^{T}$ and $\mathbf{t}_{3} \mathbf{t}_{3}^{T}$, should be considered independently using the same procedure. Thus, the conditions for the three joints to realize a given $\mathbf{C}$ are:

$$
\begin{aligned}
& \left(\mathbf{r}_{1}^{T} \boldsymbol{\Omega} \mathbf{r}_{2} \mathbf{r}_{2}^{T} \mathbf{C r}_{1}\right)\left(\mathbf{r}_{1}^{T} \boldsymbol{\Omega} \mathbf{r}_{3} \mathbf{r}_{3}^{T} \mathbf{C r}_{1}\right) \leq 0 \\
& \left(\mathbf{r}_{2}^{T} \boldsymbol{\Omega} \mathbf{r}_{3} \mathbf{r}_{3}^{T} \mathbf{C r}_{2}\right)\left(\mathbf{r}_{2}^{T} \boldsymbol{\Omega} \mathbf{r}_{1} \mathbf{r}_{1}^{T} \mathbf{C r}_{2}\right) \leq 0 \\
& \left(\mathbf{r}_{3}^{T} \boldsymbol{\Omega} \mathbf{r}_{1} \mathbf{r}_{1}^{T} \mathbf{C} \mathbf{r}_{3}\right)\left(\mathbf{r}_{3}^{T} \boldsymbol{\Omega} \mathbf{r}_{2} \mathbf{r}_{2}^{T} \mathbf{C r}_{3}\right) \leq 0
\end{aligned}
$$

Note that these conditions are not independent. If any two of the three inequalities hold, then the remaining inequality must be true.

Proposition 2: An arbitrary compliance $\mathbf{C}$ can be realized with three compliant joints with positions $\mathbf{r}_{1}, \mathbf{r}_{2}$ and $\mathbf{r}_{3}$ if and only if any two inequalities of (13)-(15) hold.

\subsection{Conditions on Configuration for Compliance Realization}

Similar to that for $J_{1}$ discussed in Section 3.1, consider the two vectors associated with $J_{2}$ given by:

$$
\tilde{\mathbf{t}}_{2}=\mathbf{C r}_{2}, \quad \tilde{\mathbf{r}}_{2}=\mathbf{\Omega} \mathbf{C r}_{2},
$$

and plane $P_{2}$ defined by the two rays $\mathbf{t}_{2} \mathbf{t}_{2}$ and $\tilde{\mathbf{t}}_{2} \tilde{\mathbf{t}}_{2}$. Since the compliance matrix $\mathbf{C}$ is on both plane $P_{1}$ and plane $P_{2}$, it must be at the intersection of the two planes as illustrated in Fig. 7 .

If $\mathbf{C}$ is realized by a three joint serial mechanism, plane $P_{1}$ must separate $\mathbf{t}_{2} \mathbf{t}_{2}^{T}$ and $\mathbf{t}_{3} \mathbf{t}_{3}^{T}$, and plane $P_{2}$ must separate $\mathbf{t}_{1} \mathbf{t}_{1}^{T}$ and $\mathbf{t}_{3} \mathbf{t}_{3}^{T}$. Inequalities (13) and (14) require that the joint compliance $\mathbf{t}_{3} \mathbf{t}_{3}^{T}$ be on the arc between $\tilde{\mathbf{t}}_{1} \tilde{\mathbf{t}}_{1}^{T}$ and $\tilde{\mathbf{t}}_{2} \tilde{\mathbf{t}}_{2}^{T}$ as shown in Fig. 7 .

Note that joint twist $\mathbf{t}_{i}$ and joint position $\mathbf{r}_{i}$ have the relationship:

$$
\mathbf{t}_{i}= \pm \Omega \mathbf{r}_{i}, \quad \tilde{\mathbf{t}}_{i}= \pm \Omega \mathbf{C r}_{i}
$$

Since the two rays associated with $\tilde{\mathbf{t}}_{1} \tilde{\mathbf{t}}_{1}^{T}$ and $\tilde{\mathbf{t}}_{2} \tilde{\mathbf{t}}_{2}^{T}$ set the bounds on $\mathbf{t}_{3} \mathbf{t}_{3}^{T}$ in $2 \times 2$ matrix space, the two vectors $\boldsymbol{\Omega} \mathbf{C r}_{1}$ and $\mathbf{\Omega} \mathbf{C r}_{2}$ define the bounds on $\mathbf{r}_{3}$ in 2 -vector space and can be represented in terms of the mechanism geometry. 


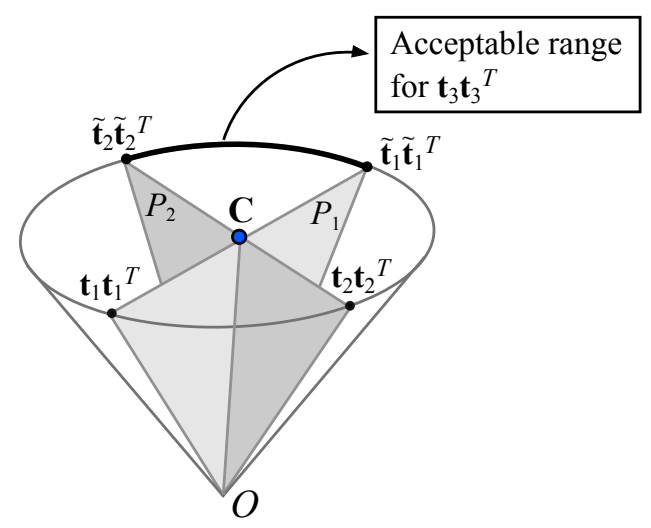

Figure 7: Locus of acceptable joint compliance of $J_{3}$. The compliance associated with joint-3, $\mathbf{t}_{3} \mathbf{t}_{3}^{T}$, must lie on the arc between $\tilde{\mathbf{t}}_{1} \tilde{\mathbf{t}}_{1}^{T}$ and $\tilde{\mathbf{t}}_{2} \tilde{\mathbf{t}}_{2}^{T}$ (which depend on the given positions of $J_{1}$ and $J_{2}$ ).

In the plane of the mechanism, consider two lines, $l_{1}$ and $l_{2}$, passing through the origin $O$ (where the compliance is specified), defined by $l_{1} \perp \mathbf{C r}_{1}$ and $l_{2} \perp \mathbf{C r}_{2}$. The two lines separate the space into four subspaces: $S_{\mathrm{I}}, S_{\mathrm{II}}, S_{\mathrm{III}}$, and $S_{\mathrm{IV}}$ as shown in Fig. 8.

It can be seen that if vector $\mathbf{r}_{3}$ satisfies conditions (13)-(14), then $-\mathbf{r}_{3}$ must also satisfy the same conditions. Thus, if inequalities (13)-(14) are true for $S_{\mathrm{I}}$, they must be also true for $S_{\mathrm{III}}$.

Denote:

$$
\begin{aligned}
& \sigma_{1}=S_{\mathrm{I}} \cup S_{\mathrm{III}}, \\
& \sigma_{2}=S_{\mathrm{II}} \cup S_{\mathrm{IV}} .
\end{aligned}
$$

Then, $\mathbf{r}_{3}$ must be either in $\sigma_{1}$ or in $\sigma_{2}$ to realize the compliance $\mathbf{C}$. In fact, if any point in $\sigma_{1}$ satisfies inequalities (13)-(14), the following must be true:

(i). Every point in $\sigma_{1}$ satisfies the same conditions;

(ii). No point inside $\sigma_{2}$ satisfies the same conditions.

The above statements (i) and (ii) can be proved by the fact that, for a given $\mathbf{r}_{1}$ and $\mathbf{r}_{2}$, the expressions on the left side of inequalities (13)-(14) are continuous functions of $\mathbf{r}_{3}$, and if one inequality changes sign, $\mathbf{r}_{3}$ must cross either line $l_{1}$ or line $l_{2}$.

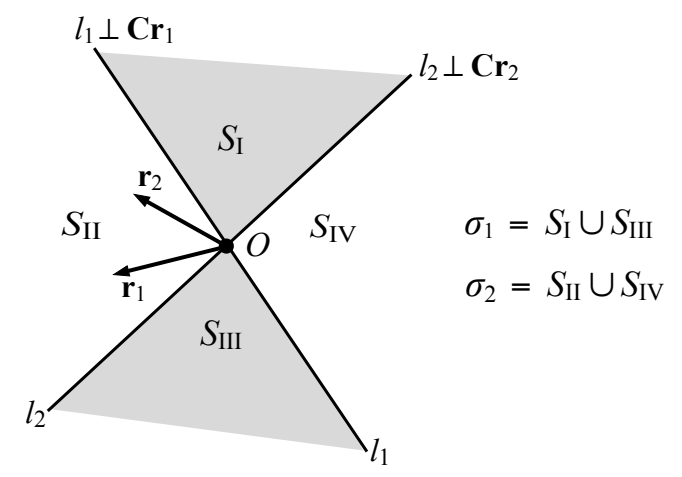

Figure 8: The acceptable space of $\mathbf{r}_{3}$ for realizing $\mathbf{C}$. The space of $\mathbf{r}_{3}$ for the realization of $\mathbf{C}$ is a zone limited by the two lines $l_{1}$ and $l_{2}, \sigma_{1}$ or $\sigma_{2}$. The space is identified by the fact: neither $\mathbf{r}_{1}$ nor $\mathbf{r}_{2}$ can be inside of the acceptable space.

Thus, once the positions of $J_{1}$ and $J_{2}$ are specified, the acceptable space for $J_{3}$ to realize $\mathbf{C}$ is a zone with vertical (opposite) angles and boundaries of $l_{1}$ and $l_{2}$, either the space $\sigma_{1}$ or the space $\sigma_{2}$ shown in 
Fig. 8. Although the space can be identified by testing conditions (13)-(14) using an arbitrary point, the space can also be identified directly by testing the position of $J_{1}$ or $J_{2}$. Neither $\mathbf{r}_{1}$ nor $\mathbf{r}_{2}$ can be in the interior of the space acceptable for $\mathbf{r}_{3}$. This can be proved by setting $\mathbf{r}_{3}=\mathbf{r}_{1}$ in condition (13), which violates the inequality. Therefore, as shown in Fig. $9, \mathbf{r}_{3}$ or $-\mathbf{r}_{3}$ must be separated from $\mathbf{r}_{1}$ by $l_{1}$. By the same reasoning, $\mathbf{r}_{3}$ or $-\mathbf{r}_{3}$ must be separated from $\mathbf{r}_{2}$ by $l_{2}$, and $\mathbf{r}_{1}$ or $-\mathbf{r}_{1}$ must be separated from $\mathbf{r}_{2}$ by line $l_{3} \perp \mathbf{C r}_{3}$. Figure 9 shows the geometric relations among position vectors $\mathbf{r}_{i}$ 's and the lines $l_{i}$ 's for a mechanism configuration that realizes a given compliance $\mathbf{C}$.

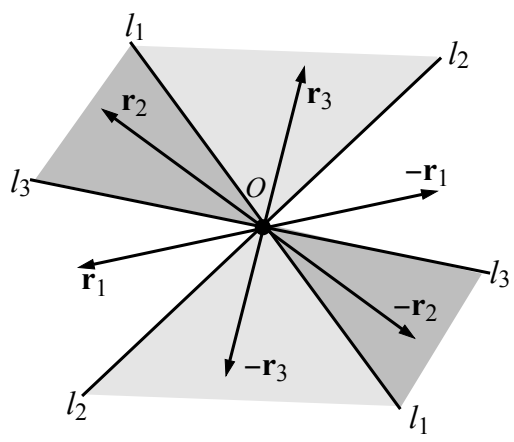

Figure 9: Necessary and sufficient condition for a compliance to be realized by a $3 \mathrm{R}$ mechanism. Position vector $\mathbf{r}_{3}$ is adjacent to $l_{1}$ and $l_{2} ; \mathbf{r}_{2}$ is adjacent to $l_{1}$ and $l_{3} ;$ and $\mathbf{r}_{1}$ is adjacent to $l_{2}$ and $l_{3}$.

In summary, we have:

Proposition 3: Consider a compliance matrix $\mathbf{C}$ and a 3R mechanism having joint positions $\mathbf{r}_{i}$. Let $l_{i}$ be a line passing through $O$ and perpendicular to vector $\mathbf{C r}_{i}(i=1,2,3)$. Then,

a). If $\mathbf{r}_{1}$ and $\mathbf{r}_{2}$ are specified, the space of $\mathbf{r}_{3}$ that can realize $\mathbf{C}$ is the zone $\sigma_{1}$ that does not contain $\mathbf{r}_{1}$ (or $\mathbf{r}_{2}$ ) as shown in Fig. 8;

b). If $\mathbf{r}_{1}, \mathbf{r}_{2}$ and $\mathbf{r}_{3}$ are specified, $\mathbf{C}$ can be realized if and only if: $\pm \mathbf{r}_{1}$ is between and adjacent to lines $l_{2}$ and $l_{3} ; \pm \mathbf{r}_{2}$ is between and adjacent to lines $l_{1}$ and $l_{3}$; and $\pm \mathbf{r}_{3}$ is between and adjacent to lines $l_{1}$ and $l_{2}$ as shown in Fig. 9.

Similar to conditions (13)-(15), the three conditions on mechanism configuration in Proposition 3b are not independent. If any two conditions in Proposition $3 \mathrm{~b}$ are true, the remaining condition must also be true.

\subsection{Example}

An application of these results is illustrated in Fig. 10. This 3R mechanism example shows how to determine the range of the locations of $J_{3}$ when the locations of the other two joints are specified.

If a desired compliance matrix is given as:

$$
\mathbf{C}=\left[\begin{array}{ll}
2 & 1 \\
1 & 4
\end{array}\right] \times 10^{-2} \mathrm{~m} / \mathrm{N},
$$

and the locations of $J_{1}$ and $J_{2}$ are specified as:

$$
\mathbf{r}_{1}=\left[\begin{array}{c}
-0.2 \\
0
\end{array}\right] \mathrm{m}, \quad \mathbf{r}_{2}=\left[\begin{array}{r}
-0.1 \\
0.1
\end{array}\right] \mathrm{m}
$$

The two vectors $\mathbf{C r}_{1}$ and $\mathbf{C r}_{2}$ are:

$$
\mathbf{C r}_{1}=\left[\begin{array}{l}
-4 \\
-2
\end{array}\right] \times 10^{-3} \mathrm{~m}^{2} / \mathrm{N}, \quad \mathbf{C r}_{2}=\left[\begin{array}{r}
-1 \\
3
\end{array}\right] \times 10^{-3} \mathrm{~m}^{2} / \mathrm{N}
$$


The two lines $l_{1} \perp \mathbf{C r}_{1}$ and $l_{2} \perp \mathbf{C r}_{2}$ each passing through point $O$ are illustrated in Fig. 10 . For this case, $\mathbf{r}_{1}$ is along the $x$-axis. The space of acceptable $\mathbf{r}_{3}$ to realize the given $\mathbf{C}$ is identified to be the two shaded areas that meet $\mathbf{r}_{1}$ only at the origin $O$.

Figure 10 illustrates the locations of the three joints in a mechanism. When $J_{1}$ and $J_{2}$ are specified, $J_{3}$ must be in the shaded area bounded by $l_{1}$ and $l_{2}$ in order to realize the given $\mathbf{C}$. Two possible acceptable configurations $J_{1} J_{2} J_{3}$ and $J_{1} J_{2} J_{3}^{\prime}$ for the given mechanism are shown.

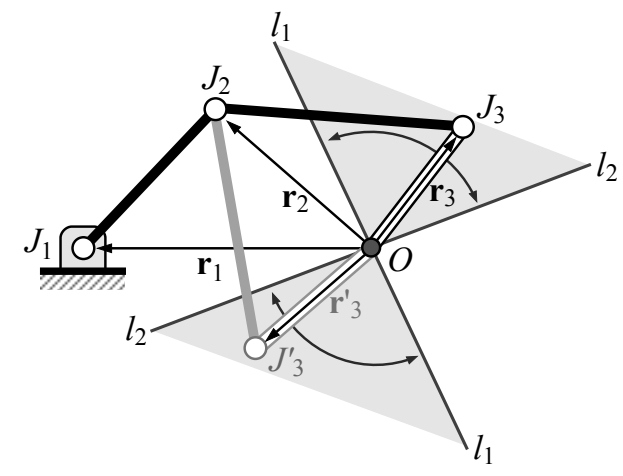

Figure 10: Configurations of a $3 \mathrm{R}$ mechanism that realizes the example compliance. Joint position vector $J_{3}$ could be anywhere in the two identified regions bounded by $l_{1}$ and $l_{2}$.

\subsection{Determination of the Joint Compliances}

Below, means for selecting the joint compliance values for a given mechanism configuration and realizable compliance $\mathbf{C}$ are derived. Unlike the calculation of Eq. (6), the matrices are not converted to vectors and a matrix inversion is not required. Only the compliance matrix and joint locations are needed.

Suppose $\mathbf{C}$ is to be realized by a $3 \mathrm{R}$ mechanism with joint twists $\mathbf{t}_{i}$, then by Eq. (5),

$$
\mathbf{C}=c_{1} \mathbf{t}_{1} \mathbf{t}_{1}^{T}+c_{2} \mathbf{t}_{2} \mathbf{t}_{2}^{T}+c_{3} \mathbf{t}_{3} \mathbf{t}_{3}^{T} .
$$

Multiplying $\mathbf{C}$ by $\mathbf{r}_{2}^{T}$ from the left and by $\mathbf{r}_{3}$ from the right yields

$$
\mathbf{r}_{2}^{T} \mathbf{C r}_{3}=c_{1} \mathbf{r}_{2}^{T} \mathbf{t}_{1} \mathbf{t}_{1}^{T} \mathbf{r}_{3}+c_{2}\left(\mathbf{r}_{2}^{T} \mathbf{t}_{2}\right) \mathbf{t}_{2}^{T} \mathbf{r}_{3}+c_{3} \mathbf{r}_{2}^{T} \mathbf{t}_{3}\left(\mathbf{t}_{3}^{T} \mathbf{r}_{3}\right) .
$$

Since $\mathbf{r}_{2}^{T} \mathbf{t}_{2}=0$ and $\mathbf{t}_{3}^{T} \mathbf{r}_{3}=0$,

$$
\mathbf{r}_{2}^{T} \mathbf{C r}_{3}=c_{1} \mathbf{r}_{2}^{T} \mathbf{t}_{1} \mathbf{t}_{1}^{T} \mathbf{r}_{3}
$$

The value for the compliance of joint-1 is:

$$
c_{1}=\frac{\mathbf{r}_{2}^{T} \mathbf{C r}_{3}}{\mathbf{r}_{2}^{T} \mathbf{t}_{1} \mathbf{t}_{1}^{T} \mathbf{r}_{3}}=\frac{\mathbf{r}_{2}^{T} \mathbf{C r}_{3}}{\mathbf{r}_{2}^{T} \Omega \mathbf{r}_{1} \mathbf{r}_{1}^{T} \boldsymbol{\Omega}^{T} \mathbf{r}_{3}} .
$$

Repeating the process for the pairs $\left(\mathbf{r}_{1}, \mathbf{r}_{3}\right)$ and $\left(\mathbf{r}_{1}, \mathbf{r}_{2}\right)$ respectively, the equations for coefficients of $c_{2}$ and $c_{3}$ are obtained. Thus, for a given configuration $\left(\mathbf{r}_{1}, \mathbf{r}_{2}, \mathbf{r}_{3}\right)$ and a realizable $\mathbf{C}$, the joint compliances are calculated using:

$$
\begin{aligned}
c_{1} & =\frac{\mathbf{r}_{2}^{T} \mathbf{C r}_{3}}{\mathbf{r}_{2}^{T} \boldsymbol{\Omega} \mathbf{r}_{1} \mathbf{r}_{1}^{T} \mathbf{\Omega}^{T} \mathbf{r}_{3}}, \\
c_{2} & =\frac{\mathbf{r}_{3}^{T} \mathbf{C} \mathbf{r}_{1}}{\mathbf{r}_{3}^{T} \boldsymbol{\Omega} \mathbf{r}_{2} \mathbf{r}_{2}^{T} \boldsymbol{\Omega}^{T} \mathbf{r}_{1}}, \\
c_{3} & =\frac{\mathbf{r}_{1}^{T} \mathbf{C} \mathbf{r}_{2}}{\mathbf{r}_{1}^{T} \boldsymbol{\Omega} \mathbf{r}_{3} \mathbf{r}_{3}^{T} \boldsymbol{\Omega}^{T} \mathbf{r}_{2}}
\end{aligned}
$$


Note that once the configuration is specified, the compliance values are each unique. As stated in Proposition 2 , if $\mathbf{C}$ is realizable, then inequalities (13)-(15) must be satisfied. It can be proved that inequalities (13)-(15) ensure that each $c_{i}$ in (18)-(20) is positive. Thus, if $\mathbf{C}$ is realizable, the coefficients calculated using Eqs. (18)-(20) are all positive. In fact, $c_{i}>0(i=1,2,3)$ is also a necessary and sufficient condition for $\mathbf{C}$ to be realized with the mechanism at the given configuration.

\subsection{Mechanisms with More Than Three Compliant Joints}

Consider a mechanism having $n(n>3)$ revolute joints. At a configuration, joint $i$ has position $\mathbf{r}_{i}$ and joint twist $\mathbf{t}_{i}, i=1,2, \ldots, n$. If a compliance $\mathbf{C}$ is realized by the mechanism at the configuration, then $\mathbf{C}$ must be in the polyhedral convex cone bounded by the edges (rays) associated with the rank-1 joint compliance $\mathbf{t}_{i} \mathbf{t}_{i}^{T}$, where each edge is on the boundary of the PSD cone.

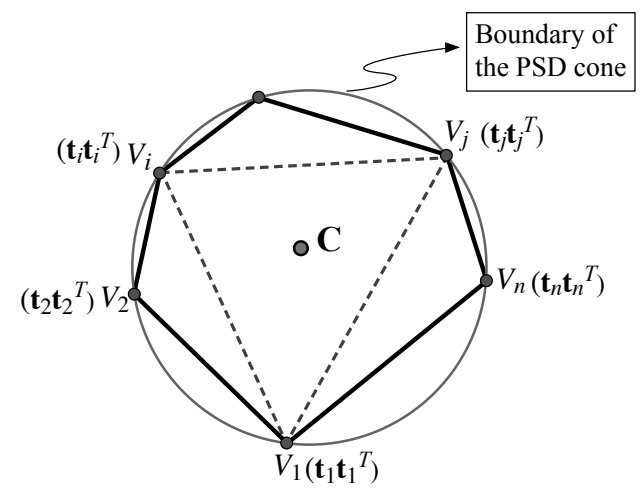

Figure 11: Cross section of the PSD cone containing the compliance C. The vertexes $V_{i}$ of the polygon are associated with joint compliance $\mathbf{t}_{i} \mathbf{t}_{i}^{T}$. C must be inside the polygon to be realized with the mechanism.

Figure 11 shows a cross section of the PSD cone containing the compliance $\mathbf{C}$. In this plane, the edges (rays) associated with the joint compliances $\mathbf{t}_{i} \mathbf{t}_{i}^{T}$ s are represented by points $V_{i}$ on the boundary of the PSD cone, and the polygon with vertexes $V_{i}$ is the cross section of the polyhedral convex cone of all realizable compliances. It can be seen that the compliance $\mathbf{C}$ can be realized with the mechanism at the configuration if and only if $\mathbf{C}$ is within the polygon with vertexes $V_{i}$.

Consider the vertex $V_{1}$ in the plane shown in Fig. 11. If $\mathbf{C}$ is in the polygon $V_{1} V_{2} \cdots V_{n}$, there must exist two vertexes $V_{i}$ and $V_{j}$ such that $\mathbf{C}$ is the triangle $V_{1} V_{i} V_{j}$. This means that the compliance can be realized by three compliant joints $J_{1}, J_{i}$ and $J_{j}$. This statement is valid for any joint. As such we have:

Proposition 4: For an $n$-R mechanism having joint positions $\mathbf{r}_{i}(i=1,2, \cdots, n, n>3)$, a compliance $\mathbf{C}$ can be realized with mechanism at the configuration if and only if the following equivalent conditions are true:

a). There exists three joints in the mechanism that realize $\mathbf{C}$;

b). For every joint $J_{i}$, there exist two joints $J_{j}$ and $J_{k}$ in the mechanism such that the three joints $\left(J_{i}, J_{j}\right.$ and $\left.J_{k}\right)$ realize $\mathbf{C}$.

Thus, for an $n$-R mechanism, to determine whether a given compliance can be realized at a given configuration, condition $4 \mathrm{a}$ or $4 \mathrm{~b}$ can be used. Since only three joints are involved for each test set, conditions (13)-(15) can be used. If the compliance is realizable, one can always choose just three joints in the mechanism to realize the behavior. The corresponding values of the three joint compliances can be calculated using Eqs. (18)-(20). It can be seen that for a realizable compliance at a configuration, the set of joints that realizes the compliance is not unique.

Note that if compliance is provided at more than three joints, there are an infinite number of solutions for the set of joint compliances that achieve a realizable compliance. A closed form solution cannot be obtained if no additional constraints are considered. 
As shown above, the kinematics of a mechanism plays a significant role in compliance realization. In the following section, mechanism geometries that facilitate realization of an arbitrary compliance are identified.

\section{Realization of Any Compliance with a Given Mechanism}

Section 3 addressed the realizability of a single given compliance for a given mechanism configuration. Since an $n$-R mechanism has $n$ degrees-of-freedom, when $n \geq 3$, the configuration can change when the position of endpoint $O$ relative to the mechanism base $J_{1}$ is specified. A compliance unrealizable with one configuration could be realizable by changing the configuration. Here, the ability of a given $n$-R mechanism to realize every particle planar compliance at its endpoint without changing the endpoint location is investigated.

First, mechanisms having three joints are considered. A requirement on the twist spaces associated with joints $J_{2}$ and $J_{3}$ is identified. Next, the requirement on the twist space is converted to geometric conditions on the mechanism. We show that if a $3 \mathrm{R}$ mechanism has appropriate link length ratios (including the distance from $J_{1}$ to $O$ ), all compliances can be realized at the given endpoint location. Then, the results for $3 \mathrm{R}$ mechanisms are extended to $n$-R mechanisms. A discussion on the workspace and the space of endpoint locations that ensure the mechanism ability to realize all compliances is presented.

\subsection{Realization Conditions for Every Compliance}

Consider a 3R serial mechanism in which the endpoint location $O$ relative to the base $J_{1}$ is specified. The mechanism can be viewed as a four-bar linkage with $J_{1} O$ grounded. The position of $J_{1}$ relative to the compliance frame, $\mathbf{r}_{1}$, is constant.

If a compliance matrix $\mathbf{C}$ is given, line $l_{1} \perp \mathbf{C r}_{1}$ is specified. When the configuration of the mechanism changes, two joints, $J_{2}$ and $J_{3}$, change their positions. The position vectors of $J_{2}$ and $J_{3}, \mathbf{r}_{2}$ and $\mathbf{r}_{3}$, span different ranges depending on the mechanism geometry. We show that the capability of a mechanism to realize an arbitrary compliance depends on the ranges spanned by $\mathbf{r}_{2}$ and $\mathbf{r}_{3}$. In fact, we have:

Proposition 5: Consider a mechanism having three compliant revolute joints with positions $\mathbf{r}_{i}$, $i=1,2,3$.

a). A given compliance $\mathbf{C}$ can be realized with the mechanism if $\mathbf{r}_{2}$ or $\mathbf{r}_{3}$ can cross line $l_{1} \perp \mathbf{C r}_{1}$;

b). Every compliance matrix can be realized with the mechanism if and only if the union of spaces spanned by $\mathbf{r}_{2}$ and $\mathbf{r}_{3}$ is no less than a half plane.

The proof of Proposition 5a is based on the fact that one can aways choose a configuration such that $\mathbf{r}_{2}$ or $\mathbf{r}_{3}$ is on line $l_{1}$. The compliance can be realized with two compliant joints: either using $J_{1}$ and $J_{2}$ with $c_{3}=0$, or using $J_{1}$ and $J_{3}$ with $c_{2}=0$. Note that the realization condition in Proposition 5 a is also necessary if link-1 cannot make a full rotation. For such case, the ranges of $\mathbf{r}_{2}$ and $\mathbf{r}_{3}$ are connected. Thus, the ranges of joint compliances $\mathbf{t}_{2} \mathbf{t}_{2}^{T}$ and $\mathbf{t}_{3} \mathbf{t}_{3}^{T}$ are connected. If neither $\mathbf{r}_{2}$ nor $\mathbf{r}_{3}$ can cross $l_{1}, \mathbf{t}_{2} \mathbf{t}_{2}^{T}$ and $\mathbf{t}_{3} \mathbf{t}_{3}^{T}$ must always be on the same side of plane $P_{1}$ defined in Eq. (11). Thus, the given $\mathbf{C}$ cannot be realized at that endpoint location.

Proposition 5b is sufficient as a direct result of Proposition 3a. If the union of ranges of $\mathbf{r}_{2}$ and $\mathbf{r}_{3}$ continuously spans more than a half plane, then any straight line is within the range. Thus, for any compliance, one of the two vectors $\mathbf{r}_{2}$ or $\mathbf{r}_{3}$ must be able to cross line $l_{1}$. To prove that Proposition $5 \mathrm{~b}$ also provides a necessary condition to realize all compliances, it can be shown that all possible joints compliances $\mathbf{C}_{i}=c_{i} \mathbf{t}_{i} \mathbf{t}_{i}^{T}$ will not fill the boundary of the PSD cone if the union of ranges of $\mathbf{r}_{2}$ and $\mathbf{r}_{3}$ does not span a half space or more. Thus there are some compliances that are not comprised of a positive combination of $\mathbf{t}_{1} \mathbf{t}_{1}^{T}, \mathbf{t}_{2} \mathbf{t}_{2}^{T}$, and $\mathbf{t}_{3} \mathbf{t}_{3}^{T}$ for all possible configurations of the mechanism having the given endpoint, which proves Proposition 5(b).

The ability of a $3 \mathrm{R}$ mechanism to realize an arbitrary compliance depends on the mobility of the mechanism, especially the ranges spanned by $\mathbf{r}_{2}$ and $\mathbf{r}_{3}$. It should be noted that while the range of $\mathbf{r}_{3}$ is determined by a single rotation angle (angle of link-3), the range spanned by $\mathbf{r}_{2}$ is not directly determined by the rotation 
angle of a single link and is, therefore, more complicated to calculate. The ability to realize any compliance can be readily assessed by evaluating the rotation range of link- 3 . If link-3 can rotate more than $180^{\circ}$, then any compliance matrix can be realized with the mechanism.

In the following, geometric conditions on a $3 \mathrm{R}$ mechanism that ensure a sufficiently large twist space are developed.

\subsection{Conditions on the Mechanism Link Lengths}

Consider the $3 \mathrm{R}$ serial mechanism shown in Fig. 12. If the position of the base $J_{1}$ relative to the end-point $O$ is specified, the system is kinematically equivalent to a four-bar mechanism. The geometry of the four-bar mechanism is characterized by the three link lengths $L_{1}, L_{2}, L_{3}$ and the distance between the base joint $J_{1}$ and the endpoint $O, L_{0}$.

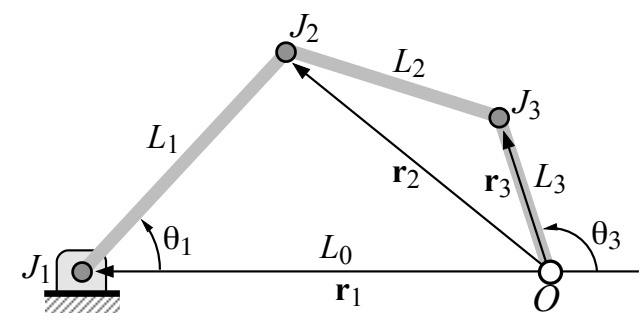

Figure 12: A 3R serial mechanism with specified endpoint. If the location of endpoint $O$ relative to base $J_{1}$ is specified, the system is kinematically equivalent to a four-bar mechanism.

It is known that if a mechanism satisfies the Grashof criterion, then at least one link is capable of turning a full rotation. For the mechanism shown in Fig. 12, if $L_{3}$ or $L_{0}$ is the shortest link, link-3 can make a full rotation [25. For this case, any compliance can be realized with the mechanism.

For a non-Grashof mechanism, none of the links can make a full rotation. Both link-1 and link-3 rock between limits but can always cross line $O J_{1}$ either clockwise or counterclockwise 25. Due to the restrictions on $\mathbf{r}_{1}$ and $\mathbf{r}_{3}$, the twist spaces associated with $J_{2}$ and $J_{3}$ are limited. However, some mechanism geometries will still allow the space spanned by $\mathbf{r}_{2}$ and $\mathbf{r}_{3}$ to be more than a half plane. It can be shown that if $L_{2}$ is the longest link, this condition is satisfied. Thus any compliance can be realized.

As stated previously, if link-3 in a mechanism can rotate more than $180^{\circ}$, then any compliance can be realized. It is known that the range of a link in a four-bar mechanism is determined by the dead points (extreme positions) of the link [25]. For the mechanism shown in Fig. 12, extreme positions of link-3 can be evaluated with minimum and maximum values of $\theta_{3}, \theta_{3}$ min and $\theta_{3}$ max.

It can be shown that if the following two conditions are satisfied

$$
\begin{aligned}
\left(L_{1}+L_{2}\right)^{2} & \geq L_{0}^{2}+L_{3}^{2}, \\
\left|L_{2}-L_{1}\right| & \leq\left|L_{0}-L_{3}\right|,
\end{aligned}
$$

then, $\theta_{3 \min } \leq 90^{\circ}$ and link-3 can rotate more than $180^{\circ}$ through $\theta_{3}=180^{\circ}$. If the following two conditions are satisfied:

$$
\begin{aligned}
\left(L_{1}-L_{2}\right)^{2} & \leq L_{0}^{2}+L_{3}^{2} \\
L_{1}+L_{2} & \geq L_{0}+L_{3}
\end{aligned}
$$

then, $\theta_{3 \max } \geq 90^{\circ}$ and link-3 can rotate more than $180^{\circ}$ through $\theta_{3}=0^{\circ}$.

In summary, we have:

Proposition 6. Consider an arbitrary $3 \mathrm{R}$ serial mechanism with specified location of endpoint $O$ relative to the base $J_{1}$. 
a). If the mechanism is Grashof with $L_{3}$ or $L_{0}$ being the shortest link, then any compliance matrix can be realized.

b). If the mechanism is non-Grashof with $L_{2}$ being the longest link, then any compliance matrix can be realized.

c). If the mechanism satisfies conditions (21)-(22) or conditions (23)-(24), then any compliance can be realized.

\subsection{Mechanisms with More Than Three Joints}

The results obtained for $3 \mathrm{R}$ mechanisms can be extended to $n$-R mechanisms $(n>3)$. Similar to Proposition 5, we have:

Proposition 7: Consider a mechanism having $n(n>3)$ compliant revolute joints. Suppose $\mathbf{r}_{i}$ is the position vector of joint $J_{i}, i=1,2, \cdots, n$.

a). A given compliance $\mathbf{C}$ can be realized with the mechanism if there is a joint $J_{i}$ that can cross line $l_{1} \perp \mathbf{C r}_{1}$;

b). Every compliance matrix can be realized with the mechanism if and only if the union of spaces spanned by $\mathbf{r}_{i}$ 's $(2 \leq i \leq n)$ is no less than a half plane.

Similar to the 3R case discussed in Section 4.2, when the mechanism base and the end-point (where the compliance frame is located) are specified, an $n$-R mechanism is kinematically equivalent to an $(n+1)$-bar linkage. Due to the increase in degrees of freedom, the description of the space spanned by each $\mathbf{r}_{i}$ is much more complicated. However, for some cases, the mechanism ability to realize all compliances can be assessed by the rotation range of the $n$th link. If link- $n$ can rotate more than $180^{\circ}$, then every compliance can be achieved with the mechanism. Below, a necessary and sufficient condition for link- $n$ to have this ability is obtained using the theory presented in [26].

Consider an $n$-R mechanism with link length $L_{i}$ as shown in Fig. 13. Suppose that the distance between the base joint $J_{1}$ and the end-point $O$ is specified as $L_{0}$.

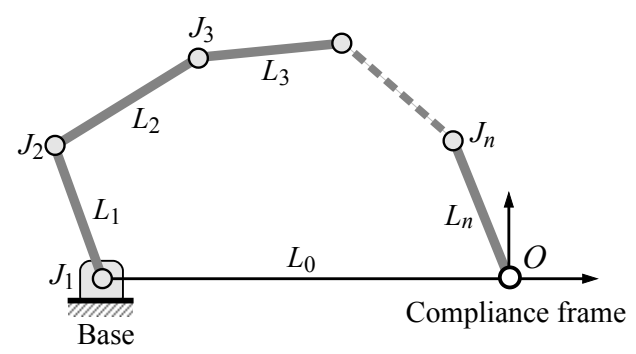

Figure 13: An $n$-R mechanism. When the mechanism base $J_{1}$ and the end-point $O$ are specified, the mechanism is kinematically equivalent to an $(n+1)$-bar linkage with $L_{0}$ grounded.

Denote:

$$
L_{i, j}=\min \left(L_{i}, L_{j}\right), \quad L_{\max }=\max \left(L_{0}, L_{1}, \cdots, L_{n}\right) .
$$

Then, the two adjacent links $L_{i}$ and $L_{j}$ can revolve relative to each other if and only if the sum of $L_{i, j}$ and $L_{\max }$ is no greater than the sum of all remaining link lengths [26]. For the two adjacent links $L_{0}$ and $L_{n}$, the condition can be expressed as:

$$
L_{\max }+\min \left(L_{n}, L_{0}\right) \leq \frac{1}{2} \sum_{i=0}^{n} L_{i} .
$$

Since $L_{0}$ is grounded, condition (25) is a necessary and sufficient condition for link- $n$ to make a full rotation. Thus, condition (25) is a sufficient condition for a mechanism to achieve all compliances. 


\subsection{Discussion}

A 3R serial mechanism is an open chain having 3 DOF. Although it is kinematically equivalent to a four-bar linkage when the endpoint $O$ (the location of the compliance frame) is specified, the endpoint is clearly not fixed in space. The implications of this analogy on the manipulator workspace are described below.

Figure 14 shows, for two different cases of manipulator geometry, both the manipulator workspace and the space of endpoint locations for which all compliances can be realized.

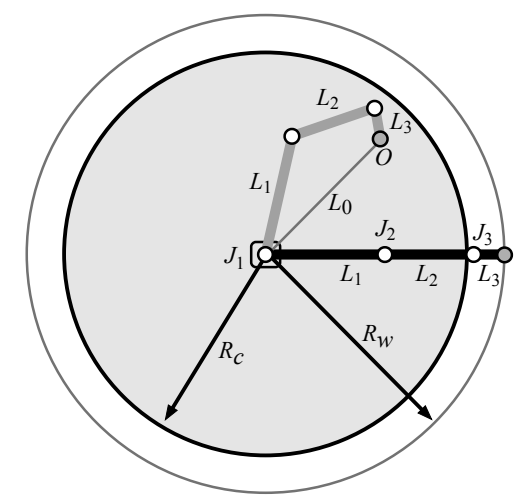

(a)

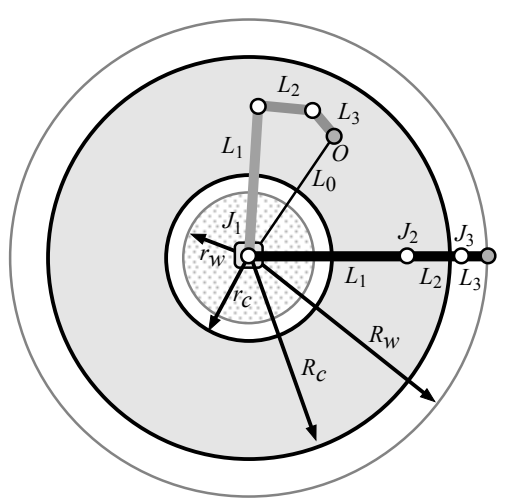

(b)

Figure 14: The workspace and the space of endpoint locations that ensure the mechanism ability to realize any compliance matrix. (a) Case 1: $L_{1}>L_{2}>L_{3}$ and $L_{2}+L_{3}>L_{1}$. (b) Case 2: $L_{1}>L_{2}>L_{3}$ and $L_{2}+L_{3}<L_{1}$.

In Fig. 14a, the link lengths of the manipulator satisfy $L_{1}>L_{2}>L_{3}$ and $L_{2}+L_{3}>L_{1}$. The manipulator workspace is a circle of radius $R_{w}=L_{1}+L_{2}+L_{3}$. It can be proved that if the location of the mechanism endpoint is inside of the circle of radius $R_{c}$, where

$$
R_{c}=\sqrt{\left(L_{1}+L_{2}\right)^{2}-L_{3}^{2}},
$$

then any compliance can be realized with the manipulator at that location.

In Fig. 14b, the link lengths of the manipulator satisfy $L_{1}>L_{2}>L_{3}$, but $L_{2}+L_{3}<L_{1}$. The manipulator workspace is an annulus between the circle of radius $R_{w}=L_{1}+L_{2}+L_{3}$ and the circle of radius $r_{w}=$ $L_{1}-L_{2}-L_{3}$. It can be proved that if the location of the endpoint is inside of the annulus between the circle of radius $R_{c}$ and the circle of radius $r_{c}$, where

$$
\begin{aligned}
R_{c} & =\sqrt{\left(L_{1}+L_{2}\right)^{2}-L_{3}^{2}}, \\
r_{c} & =\sqrt{\left(L_{1}-L_{2}\right)^{2}-L_{3}^{2}},
\end{aligned}
$$

then any compliance can be realized with the manipulator at that location.

It can be seen that, for both cases, if link-3 is short, the space of endpoint locations for which all compliances can be realized is close to the mechanism workspace.

\section{Synthesis of a Realizable Compliance}

If it is possible to realize a compliance at a given location with a given mechanism, a procedure for finding an appropriate configuration and associated joint compliance coefficients is needed. In this section, two types of synthesis procedures for any given realizable compliance are provided. First, a synthesis procedure for the realization of a compliance using a $3 \mathrm{R}$ mechanism having only two compliant joints is developed. Then, 
a synthesis procedure for the general case in which all three joints of a $3 \mathrm{R}$ mechanism are compliant is presented.

The synthesis procedures presented in this section for $3 \mathrm{R}$ mechanisms can be applied to $n$-R mechanisms using the results presented in Section 3.5.

\subsection{Synthesis with a Mechanism Having Two Compliant Joints}

Since a non-singular $2 \times 2$ compliance $\mathbf{C}$ is rank 2 , to realize a given $\mathbf{C}$ at least two compliant joints are required. Proposition 4 shows that if a compliance $\mathbf{C}$ can be realized with a $3 \mathrm{R}$ mechanism, $\mathbf{C}$ can always be realized with only two compliant joints, either $J_{1}$ and $J_{2}$ or $J_{1}$ and $J_{3}$, with the remaining joint having $c_{i}=0$. If a mechanism satisfies the conditions in Propositions 6 and 7 , then any compliance can be realized with joints $J_{1}$ and $J_{3}$ alone. For these mechanisms, adding compliance to the intermediate joint (joint-2) does not enlarge the realizable compliance space of the mechanisms.

Although loading all three joints with compliance is not necessary to realize a given compliance, mechanisms with three compliant joints have the advantage that a significant amount of compliances (a large "neighborhood" in the PSD space) can be reached with the mechanism by just changing the joint compliances while keeping the configuration unchanged. For a mechanism with only two compliant joints, when a compliance varies in the realizable space, both the mechanism configuration and the joint compliances need to be changed.

Below a synthesis procedure is developed to realize an arbitrary compliance with a 3R mechanism having compliance at joints $J_{1}$ and $J_{3}$. For a given compliance $\mathbf{C}$, the procedure determines the mechanism configuration and the joint compliances.

Consider a given mechanism having link lengths $L_{1}, L_{2}$, and $L_{3}$ as shown in Fig. 12 . The distance between the base joint $J_{1}$ and compliance frame origin is specified as $L_{0}$.

A $2 \times 2$ compliance matrix $\mathbf{C}$ to be realized is expressed in the coordinate frame $O x y$ having the form:

$$
\mathbf{C}=\left[\begin{array}{ll}
a & b \\
b & d
\end{array}\right] \text {. }
$$

In the coordinate frame $O x y$, the position of $J_{1}$ and the corresponding twists are:

$$
\mathbf{r}_{1}=\left[\begin{array}{c}
-L_{0} \\
0
\end{array}\right] \text { and } \mathbf{t}_{1}=\left[\begin{array}{c}
0 \\
L_{0}
\end{array}\right]
$$

The 3-step synthesis procedure is:

1. Decompose $\mathbf{C}$ into two rank-1 matrices:

$$
\begin{aligned}
\mathbf{C} & =\left[\begin{array}{ll}
a & b \\
b & d
\end{array}\right]=\left[\begin{array}{cc}
0 & 0 \\
0 & d-\frac{b^{2}}{a}
\end{array}\right]+\left[\begin{array}{cc}
a & b \\
b & \frac{b^{2}}{a}
\end{array}\right] \\
& =\mathbf{C}_{1}+\mathbf{C}_{3} .
\end{aligned}
$$

Since $\mathbf{C}$ is a PSD matrix, $a>0, d>0$, and

$$
a d-b^{2} \geq 0 \quad \Longrightarrow \quad d-\frac{b^{2}}{a} \geq 0,
$$

and $\mathbf{C}_{1}$ and $\mathbf{C}_{3}$ are PSD matrices.

2. Synthesize $\mathbf{C}_{1}$ with compliant joint $J_{1}$.

Choose

$$
c_{1}=\frac{d-\frac{b^{2}}{a}}{L_{0}^{2}}>0,
$$

then, $\mathbf{C}_{1}=c_{1} \mathbf{t}_{1} \mathbf{t}_{1}^{T}$. 
3. Synthesize $\mathbf{C}_{3}$ with compliant joint $J_{3}$.

Express $\mathbf{C}_{3}$ in dyad form:

$$
\mathbf{C}_{3}=\left[\begin{array}{cc}
a & b \\
b & \frac{b^{2}}{a}
\end{array}\right]=\left[\begin{array}{c}
\sqrt{a} \\
\frac{b}{\sqrt{a}}
\end{array}\right]\left[\begin{array}{ll}
\sqrt{a}, & \frac{b}{\sqrt{a}}
\end{array}\right] .
$$

- Calculate angle $\theta_{3}$.

The slope of the vector $\mathbf{C r}_{1}$ is $\frac{b}{a}$. In order for link-3 to be on line $l_{1} \perp \mathbf{C r}_{1}$,

$$
\theta_{3}=\tan ^{-1} \frac{b}{a} \pm 90^{\circ}
$$

- Determine the joint compliance $c_{3}$.

The joint compliance can be calculated by:

$$
c_{3}=\frac{a+\frac{b^{2}}{a}}{L_{3}^{2}} .
$$

Then, $\mathbf{C}_{3}=c_{3} \mathbf{t}_{3} \mathbf{t}_{3}^{T}$.

With the final step, the mechanism configuration and joint compliances are determined. It is noted that Eq. (27) yields two configurations. Each realizes the same compliance $\mathbf{C}$.

\subsection{Synthesis with a Mechanism Having Three Compliant Joints}

A synthesis procedure is developed to realize a given compliance $\mathbf{C}$ in which all three joints of a $3 \mathrm{R}$ mechanism are compliant. This development is based on the procedure used for the 2-compliant joint case (described in Section 5.1).

When the position of the endpoint relative to the joint base $J_{1}$ is specified, the position vector $\mathbf{r}_{1}$ is constant and line $l_{1} \perp \mathbf{C r}_{1}$ is determined. Since the mechanism has one DOF, the locations of $J_{2}$ and $J_{3}$ are related by the geometry of a four-bar linkage. In order to ensure $J_{3}$ is in an acceptable position, the process presented in Section 4.1 can be used to estimate the acceptable space for $\mathbf{r}_{3}$. First, consider the configuration that realizes the given compliance $\mathbf{C}$ using two compliant joints $J_{1}$ and $J_{3}$. As shown in 4.1, the position of $J_{2}, \mathbf{r}_{2}^{\prime}$, and line $l_{2}^{\prime} \perp \mathbf{C r}_{2}^{\prime}$ are obtained. The two lines $l_{1}$ and $l_{2}^{\prime}$ can be used to estimate the acceptable space of $\mathbf{r}_{3}$ based on Proposition 3 a. A location for $J_{3}$ can be chosen in the acceptable space.

The 4-step synthesis procedure is:

1. Calculate the vector $\mathbf{C r}_{1}$ and determine line $l_{1} \perp \mathbf{C r}_{1}$.

2. Choose an angle of $\mathbf{r}_{3}$ (or a location of $J_{3}$ ) such that $\mathbf{r}_{3}$ is in an acceptable zone described in Section 3.2 .

The angle $\theta_{3}$ can be found through the following:

(a) Evaluate the dead points of link-3 and determine the rotation range of $\theta_{3}$.

(b) Calculate the angle $\theta_{3}^{\prime}$ corresponding to line $l_{1}$ using Eq. (27).

(c) Calculate the position of $J_{2}, \mathbf{r}_{2}^{\prime}$, assuming $\theta_{3}=\theta_{3}^{\prime}$.

(d) Determine the line $l_{2}^{\prime} \perp \mathbf{C r}_{2}^{\prime}$.

(e) Choose $\mathbf{r}_{3}$ in the zone bounded by $l_{1}$ and $l_{2}^{\prime}$. The zone is identified using Proposition 3a.

The angle $\theta_{3}$ associated with $\mathbf{r}_{3}$ is now determined.

3. Calculate $\mathbf{r}_{2}$ associated with the configuration yielding $\theta_{3}$.

4. Determine the joint compliance constants using Eqs. (18)-(20) presented in Section 3.4.

With the final step the configuration and joint compliances of the mechanism are determined. It is noted that when $\mathbf{r}_{2}$ is obtained in Step 3, line $l_{2} \perp \mathbf{C r}_{2}$ needs to be constructed to confirm that $\mathbf{r}_{3}$ is between $l_{1}$ and $l_{2}$ and satisfies the condition in Proposition 3a. If the conditions are not satisfied, a new position of $\mathbf{r}_{3}$ closer to $l_{1}$ should be selected. It is also noted that the synthesis solution is not unique.

A numerical example is used to to illustrate the synthesis procedures in Section 6. 


\section{Synthesis Procedure Examples}

An example is provided to illustrate the synthesis procedures presented in Section 5. The compliance matrix to be realized is the same $\mathbf{C}$ used in Section 3.3:

$$
\mathbf{C}=\left[\begin{array}{ll}
2 & 1 \\
1 & 4
\end{array}\right] \times 10^{-2} \mathrm{~m} / \mathrm{N}
$$

A manipulator to be used for the realization of $\mathbf{C}$ is shown in Fig. 15. The link lengths of the mechanism are given as: $L_{1}=0.4 \mathrm{~m}, L_{2}=0.3 \mathrm{~m}, L_{3}=0.2 \mathrm{~m}$. The distance between the base joint $J_{1}$ and the compliance frame origin $O$ is specified as $L_{0}=0.6 \mathrm{~m}$. Since the mechanism geometry satisfies conditions (21)-(22), any compliance can be realized by the manipulator at this endpoint location.

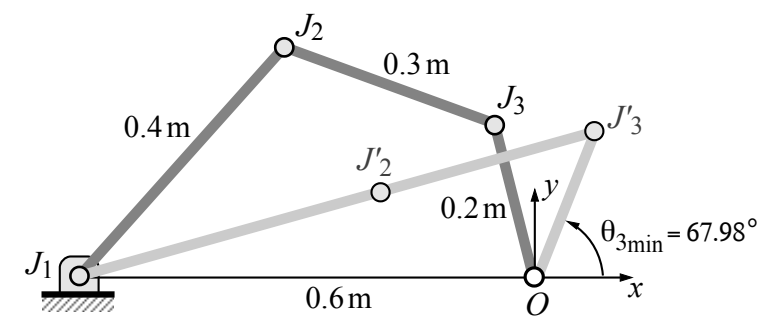

Figure 15: A specified 3R mechanism having given link lengths $L_{1}, L_{2}$ and $L_{3}$. The position of joint base $J_{1}$ relative to the compliance frame origin $O$ is specified. At an extreme configuration $J_{1} J_{2}^{\prime} J_{3}^{\prime} O, \theta_{3} \min =67.98^{\circ}$.

With the mechanism geometry, the minimum angle of link- 3 is determined to be $\theta_{3 \min }=67.98^{\circ}$. The extreme position of the mechanism is also shown in Fig. 15.

Since the synthesis of $\mathbf{C}$ using all three compliant joints is based on line $l_{1}$ and line $l_{2}^{\prime}$ obtained from the configuration at which the compliance is realized with two compliant joints $J_{1}$ and $J_{3}$, the synthesis of $\mathbf{C}$ with these two compliant joints is first performed. Then, the synthesis of $\mathbf{C}$ using all three compliant joints of the mechanism is presented.

\subsection{Synthesis of $\mathbf{C}$ with Two Compliant Joints}

The procedure presented in Section 5.1 is first used to synthesize compliance matrix $\mathbf{C}$.

Using Eq. (26), the joint compliance $c_{1}$ for $J_{1}$ is determined:

$$
c_{1}=\frac{d-\frac{b^{2}}{a}}{L_{0}^{2}}=\frac{(4-1 / 2) \times 10^{-2}}{0.6^{2}}=0.0972 \mathrm{~m} / \mathrm{N}
$$

Using Eqs. (27)-(28), the angle $\theta_{3}$ and the joint compliance $c_{3}$ for $J_{3}$ are determined:

$$
\begin{aligned}
& \theta_{3}=\tan ^{-1} \frac{b}{a} \pm 90^{\circ}=116.6^{\circ} \text { or }-63.4^{\circ}, \\
& c_{3}=\frac{a+\frac{b^{2}}{a}}{L_{3}^{2}}=\frac{(2+1 / 2) \times 10^{-2}}{0.2^{2}}=0.625 \mathrm{~m} / \mathrm{N} .
\end{aligned}
$$

If $\theta_{3}=116.6^{\circ}$ is chosen, the configuration of the mechanism is determined. Using the four-bar kinematic relations, the angle $\theta_{1}$ is calculated to be $52.38^{\circ}$. The configuration of the mechanism is illustrated in Fig. 16 . Line $l_{2}^{\prime} \perp \mathbf{C r}_{2}$ associated with the configuration is also illustrated in the figure. This line is needed in the synthesis with three compliant joints described below. 


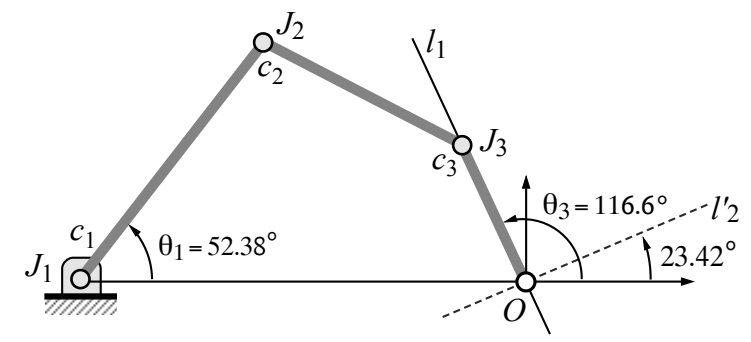

Figure 16: Configuration of the mechanism: Synthesis of of $\mathbf{C}$ with two compliant joints $J_{1}$ and $J_{3}$. The joint compliances are $c_{1}=0.0972 \mathrm{~m} / \mathrm{N}, c_{3}=0.125 \mathrm{~m} / \mathrm{N}$, and $c_{2}=0 \mathrm{~m} / \mathrm{N}$.

\subsection{Synthesis of $\mathbf{C}$ with Three Compliant Joints}

Following the procedure presented in Section 5.2, the configuration of the mechanism and joint compliances needed to realize the compliance are identified.

1. Calculate $\mathbf{C r}_{1}$ and determine line $l_{1} \perp \mathbf{C r}_{1}$, as described in Section 5.1.

2. Choose a position of joint-3 such that $\mathbf{r}_{3}$ is in the realizable zone between $l_{1}$ and $l_{2}^{\prime}$. An angle of link-3, $\theta_{3}$, can be selected based on the following conditions:

(a) The extreme position is $\theta_{3 \mathrm{~min}}=67.98^{\circ}$.

(b) The angle $\theta_{3}^{\prime}$ associated with $l_{1}$ is calculated in Section 6.1 as $\theta_{3}^{\prime}=116.6^{\circ}$.

(c) The position of $J_{2}$ associated with $\theta_{3}=116.6^{\circ}$ is calculated as $\mathbf{r}_{2}^{\prime}=[-0.3559 \mathrm{~m}, 0.3169 \mathrm{~m}]^{T}$.

(d) Line $l_{2}^{\prime} \perp \mathbf{C r}_{2}^{\prime}$ is determined. The angle between the line and the $x$-axis is $23.42^{\circ}$ (shown in Fig. 16).

(e) Choose a position between $l_{1}$ and $l_{2}^{\prime}$ using Proposition 3a that also satisfies kinematics constraints (greater than $\theta_{3 \text { min }}$ ). Here, line $l_{2}^{\prime}$ is used to estimate the acceptable zone of $\mathbf{r}_{3}$. It can be seen that the configuration $\theta_{3}=90^{\circ}$ satisfies the above conditions and is therefore chosen to realize the compliance.

3. Determine the positions of the joints.

For $\theta_{3}=90^{\circ}$, the positions of three joints are determined by the kinematics of a four-bar mechanism. The joint positions are:

$$
\mathbf{r}_{1}=\left[\begin{array}{c}
-0.6 \mathrm{~m} \\
0
\end{array}\right], \quad \mathbf{r}_{2}=\left[\begin{array}{r}
-0.2943 \mathrm{~m} \\
0.2580 \mathrm{~m}
\end{array}\right], \quad \mathbf{r}_{3}=\left[\begin{array}{c}
0 \\
0.2 \mathrm{~m}
\end{array}\right]
$$

4. Determine the joint compliance coefficients using Eqs. (18)-(20).

$$
\begin{aligned}
& c_{1}=0.0794 \mathrm{~m} / \mathrm{N}, \\
& c_{2}=0.1317 \mathrm{~m} / \mathrm{N}, \\
& c_{3}=0.2809 \mathrm{~m} / \mathrm{N} .
\end{aligned}
$$

With the final step, the mechanism configuration with $\theta_{3}=90^{\circ}$ and the joint compliances are obtained. The mechanism configuration is illustrated in Fig. 17. To confirm that the mechanism configuration satisfies the realization conditions, line $l_{2} \perp \mathbf{C r}_{2}$ corresponding to the final configuration is also illustrated in the figure.

Note that, when synthesizing a given compliance with two compliant joints $J_{1}$ and $J_{3}$, the configuration and joint compliance of the mechanism are uniquely determined; when synthesizing a given compliance with three compliant joints, the configuration and joint compliance of the mechanism are non-unique. There are an infinite number of configurations and sets of joint compliances for the given mechanism that realize the same compliance. 


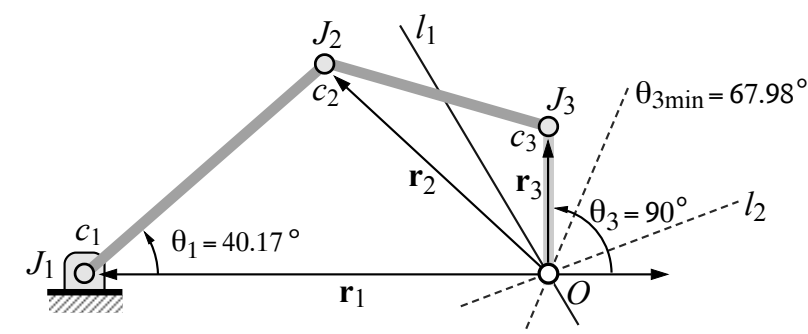

Figure 17: Configuration of the mechanism: Synthesis of of $\mathbf{C}$ with three compliant joints $J_{1}, J_{2}$, and $J_{3}$.

\section{Discussion and Conclusions}

The decomposition process presented in the paper is theoretical. For a given Cartesian compliance, the process yields the mechanism configuration and the joint compliances. In this paper, we assume that any set of joint compliances can be attained via a conventional (passive) spring at each joint, or SEAs/VSAs. In practical application, the prescribed global compliance matrix will be influenced by the following factors: 1) gravity or loading of joint compliances changes the mechanism equilibrium and causes non-symmetry of the global compliance; 2) the range of the joint spring stiffness or SEA/VSA stiffness may be limited which would limit the space of global compliances achieved by the mechanism. The influence of these factors is negligible if the overall mechanism stiffness is sufficiently high and the range of joint compliances is sufficiently large.

In this paper, methods to realize an arbitrary $2 \times 2$ elastic behavior using serial mechanisms having only revolute joints are presented. The ability of any specified $3 \mathrm{R}$ mechanism to realize an arbitrary compliance behavior is characterized. It is shown that if a mechanism has appropriately sized relative link lengths, every compliance matrix can be realized by the mechanism at the specified endpoint location. This ability allows one to realize all particle compliant behaviors with a single mechanism by properly selecting the joint compliances and the mechanism configuration. Geometric appreciation of compliances associated with a mechanism provides insight into the design of manipulators that realize desired elastic behaviors. In robotic applications, a time-varying compliance can be achieved with a mechanism with VSAs by properly changing the mechanism configuration and adjusting the joint compliances.

\section{Acknowledgement}

This work is supported by the National Science Foundation under Grant IIS-1427329.

\section{References}

[1] S. Huang, J. M. Schimmels, The bounds and realization of spatial stiffnesses achieved with simple springs connected in parallel, IEEE Transactions on Robotics and Automation 14 (3) (1998) 466-475.

[2] S. Huang, J. M. Schimmels, Achieving an arbitrary spatial stiffness with springs connected in parallel, ASME Journal of Mechanical Design 120 (4) (1998) 520-526.

[3] S. Huang, J. M. Schimmels, The bounds and realization of spatial compliances achieved with simple serial elastic mechanisms, IEEE Transactions on Robotics and Automation 16 (1) (2000) 99-103.

[4] S. Huang, J. M. Schimmels, The duality in spatial stiffness and compliance as realized in parallel and serial elastic mechanisms, ASME Journal of Dynamic Systems, Measurement, and Control 124 (1) (2002) 76-84.

[5] G. Pratt, M. Williamson, Series elastic actuators, in: Proceeding of the 1995 IEEE/RSJ International Conference on Intelligent Robots and Systems, Vol. 1, Pittsburgh, PA, 1995, pp. 399-406.

[6] R. V. Ham, T. G. Sugar, B. Vanderborght, K. W. Hollander, D. Lefeber, Compliant actuator designs: Review of actuators with passive adjustable compliance/controllable stiffness for robotic applications, IEEE Robotics and Automation Magazine 16 (3) (2009) 81-94.

[7] R. S. Ball, A Treatise on the Theory of Screws, London, U.K.: Cambridge University Press, 1900.

[8] F. M. Dimentberg, The Screw Calculus and its Applications in Mechanics, Foreign Technology Division, Wright-Patterson Air Force Base, Dayton, Ohio. Document No. FTD-HT-23-1632-67, 1965.

[9] M. Griffis, J. Duffy, Kinestatic control: A novel theory for simultaneously regulating force and displacement, ASME Journal of Mechanical Design 113 (4) (1991) 508-515. 
[10] T. Patterson, H. Lipkin, Structure of robot compliance, ASME Journal of Mechanical Design 115 (3) (1993) 576-580.

[11] T. Patterson, H. Lipkin, A classification of robot compliance, ASME Journal of Mechanical Design 115 (3) (1993) $581-584$.

[12] J. Loncaric, Normal forms of stiffness and compliance matrices, IEEE Journal of Robotics and Automation 3 (6) (1987) $567-572$.

[13] R. G. Roberts, Minimal realization of a spatial stiffness matrix with simple springs connected in parallel, IEEE Transactions on Robotics and Automation 15 (5) (1999) 953-958.

[14] N. Ciblak, H. Lipkin, Synthesis of cartesian stiffness for robotic applications, in: Proceedings of the IEEE International Conference on Robotics and Automation, Detroit, MI, 1999, pp. 2147-2152.

[15] K. Choi, S. Jiang, Z. Li, Spatial stiffness realization with parallel springs using geometric parameters, IEEE Transactions on Robotics and Automation 18 (3) (2002) 264-284.

[16] M. B. Hong, Y. J. Choi, Screw system approach to physical realization of stiffness matrix with arbitrary rank, ASME Journal of Mechanisms and Robotics 1 (2) (2009) 0210071-0210078.

[17] S. Huang, J. M. Schimmels, The eigenscrew decomposition of spatial stiffness matrices, IEEE Transactions on Robotics and Automation 16 (2) (2000) 146-156.

[18] S. Huang, J. M. Schimmels, A classification of spatial stiffness based on the degree of translational-rotational coupling, ASME Journal of Mechanical Design 123 (3) (2001) 353-358.

[19] R. G. Roberts, Minimal realization of an arbitrary spatial stiffness matrix with a parallel connection of simple springs and complex springs, IEEE Transactions on Robotics and Automation 16 (5) (2000) 603-608.

[20] A. Albu-Schaffer, M. Fischer, G. Schreiber, F. Schoeppe, G. Hirzinger, Soft robotics: What cartesian stiffness can we obtain with passively compliant, uncoupled joints?, in: Proceeding of 2004 IEEE/RSJ International Conference on Intelligent Robots and Systems, Sendal, Japan, 2004, pp. 3295-3301.

[21] F. P. Petit, Analysis and control of variable stiffness robots, Ph.D. thesis, ETH Zurich, Zurich, Switzerland (2014).

[22] N. P. Belfiore, M. Verotti, P. D. Giamberardino, I. J. Rudas, Active joint stiffness regulation to achieve isotropic compliance in the euclidean space, ASME Journal of Mechanisms and Robotics 4 (4) (2012) 0410101-04101011.

[23] M. Verotti, P. Masarati, M. Morandini, N. Belfiore, Isotropic compliance in the special euclidean group SE(3), Mechanism and Machine Theory 98 (2016) 263-281.

[24] M. Verotti, N. P. Belfiore, Isotropic compliance in E(3): Feasibility and workspace mapping, ASME Journal of Mechanisms and Robotics (2015) doi: 10.1115/1.4032408.

[25] J. M. McCarthy, Geometric Design of Linkages, Springer, 2000.

[26] K. Ting, Y. Liu, Rotatability laws for $n$-bar kinematic chains and their proof, ASME Journal of Mechanical Design 113 (1) (1991) 32-39. 